\title{
A cGAMP-containing hydrogel for prolonged SARS-CoV-2 RBD subunit vaccine exposure induces a broad and potent humoral response
}

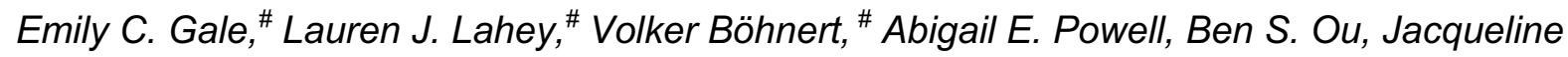
A. Carozza, Lingyin Li, ${ }^{*}$ Eric A. Appel*

E. C. Gale, Dr. E. A. Appel

Department of Materials Science and Engineering, Stanford University, Stanford, CA 94305, USA

E. C. Gale, Dr. A. E. Powell, Dr. V. Böhnert, Dr. L. Li

Department of Biochemistry, Stanford University School of Medicine, Stanford, CA 94305, USA

L. J. Lahey

Biophysics Program, Stanford University School of Medicine, Stanford, CA 94305, USA

B. S. Ou, Dr. E. A. Appel

Department of Bioengineering, Stanford University, Stanford, CA 94305

\section{J. A. Carozza}

Department of Chemistry, Stanford University, Stanford, CA 94305

L. J. Lahey, Volker Böhnert, A. E. Powell, J. A. Carozza, Dr. L. Li, Dr. E. A. Appel

Stanford ChEM-H Program, Stanford University, Stanford, CA 94305, USA

Dr. E. A. Appel

Department of Bioengineering, Stanford University, Stanford, CA 94305, USA

\# These authors contributed equally to this work

* To whom correspondence should be addressed:

eappel@stanford.edu, lingyinl@stanford.edu

\section{Keywords}

COVID-19, vaccines, STING, drug delivery, SARS-CoV-2 


\section{Abstract}

The SARS-CoV-2 virus spike protein, specifically its receptor binding domain (RBD), has emerged as a promising target for generation of neutralizing antibodies. Although the RBD peptide subunit is easily manufactured and highly stable, RBD-based subunit vaccines have been hampered by its poor inherent immunogenicity. We hypothesize that this limitation can be overcome by sustained co-administration alongside a potent and optimized adjuvant. The innate immune second messenger, cGAMP, holds promise as it activates the potent anti-viral STING pathway, but has exhibited poor performance as a therapeutic due to its nonspecific pharmacodynamic profiles when administered systemically and its poor pharmacokinetics arising from rapid excretion and degradation by its hydrolase ENPP1. To overcome these limitations, we sought to mimic the natural scenario of viral infections by creating an artificial immunological niche that enables slow release of cGAMP and the RBD antigen. Specifically, we co-encapsulated cGAMP and RBD in an injectable polymer-nanoparticle (PNP) hydrogel system. This cGAMP-adjuvanted hydrogel vaccine elicited more potent, durable, and broad antibody responses and improved neutralization than both dose-matched bolus controls and a hydrogel-based vaccine lacking cGAMP. The cGAMP-adjuvanted hydrogel platform developed is suitable for delivery of other antigens and may provide enhanced immunity against a broad range of pathogens. 


\section{Introduction}

Subunit vaccines with recombinant protein antigens are a safe and scalable approach for preventing infectious disease.$^{[1]}$ In the context of a pandemic, such as the COVID-19 pandemic, notable advantages of subunit vaccines include their favorable safety profile, high stability at warmer temperatures, and ease of manufacture at existing facilities across the globe. ${ }^{[2]}{ }^{[3]}{ }^{[4]}$ With respect to SARS-CoV-2, the receptor-binding domain (RBD) of the spike protein on the viral surface is a critical epitope required for engaging host cells to initiate infection and is therefore a promising candidate antigen. RBD is easily and efficiently produced (up to 100-fold higher expression compared to spike trimer), is relatively stable, and is the target for $90 \%$ of serum neutralizing activity in humans. ${ }^{[2]}[5][6]\left[{ }^{[7]}[8]\right.$ Unfortunately, RBD is poorly immunogenic and must be delivered with one or more adjuvant(s) to elicit an effective immune response. Moreover, while there has been progress towards drug delivery solutions for subunit vaccines to focus on improving the spatiotemporal delivery of vaccine components to the immune system, very few platforms exist that enable co-delivery of diverse antigens and adjuvant molecules, even though both sustained co-delivery and the inclusion of potent adjuvants are known to boost immune responses. ${ }^{[9]}$ There is a critical need for development of potent adjuvant systems affording spatiotemporal control over vaccine exposure to enhance immune responses to poorly immunogenic subunit antigens such as the SARS-CoV-2 RBD protein.

2'3'-cyclic-GMP-AMP (cGAMP) is a molecular second messenger that acts as a potent agonist of the stimulator of interferon genes (STING) receptor resulting in upregulation of type I interferons (IFN) and defense responses. ${ }^{[10]}$ Endogenous cGAMP production can result from diverse cellular threats, such as viral infection and cancer, and plays a powerful role in activating innate immunity. ${ }^{[11]}$ Several innate and adaptive immune cells have been described to either directly or indirectly respond to cGAMP, including subsets of NK cells, macrophages, and dendritic cells (DCs), ultimately boosting elicitation of downstream adaptive immune responses to neutralize the cellular threat. ${ }^{[12]}[13][13-15]$ Given the roles of endogenous cGAMP, using exogenous cGAMP as a therapeutic represents a promising strategy to adjuvant 
vaccines and cancer immunotherapies. However, cGAMP, as a second messenger, is rapidly degraded by its hydrolase ENPP1, which is prevalent in the plasma. ${ }^{[12]}{ }^{[13]}$ To overcome its poor stability, intratumoral injection of the non-hydrolyzable cGAMP analog ADU-S100 entered clinical trials (NCT03172936, NCT03937141) for its ability to increase tumor immunogenicity and synergize with checkpoint inhibitors. However, the non-hydrolyzable cGAMP did not deliver satisfying clinical results partially because of its poor pharmacokinetics and pharmacodynamics. At low injection concentrations, it rapidly diffuses from the site of administration causing a lack of efficacy at the desired tumor site. ${ }^{[16]}$ While injection of a high concentration of a non-degradable cGAMP analog represents one strategy to temporarily elevate the concentration at the injection site, this approach is associated with dose-dependent systemic toxicity, and adverse effects. ${ }^{[17]}$ Employing non-hydrolyzable cGAMP analogs as effective and safe vaccine adjuvants is even more challenging due to the lack of an immunological niche equivalent to the tumor microenvironment, as well as their poor pharmacokinetics and pharmacodynamics. ${ }^{[18]}\left[{ }^{19]}\right.$ We propose to harness natural cGAMP as a safe and effective adjuvant using a hydrogel depot technology that localizes and sustains low but steady cGAMP and antigen concentrations which also serves as an immunological niche; any cGAMP leaked out of the niche should be rapidly degraded by ENPP1 without causing any systemic interferon responses.

Many efforts to control cGAMP delivery have focused on engineering particle-based systems that passively drain to lymph nodes where they are taken up by cells. $\mathrm{pH}$-responsive polymerosomes, ${ }^{[20]}$ acetylated dextran microparticles, ${ }^{[21]}$ polymer nanoparticles (e.g. polyethylenimine/hyaluronic acid; poly(beta-amino ester), ${ }^{[22]}{ }^{[23]}$ and engineered pulmonary surfactant-biomimetic liposomes ${ }^{[24]}$ are all methods that have been pursued with differing success. While these cGAMP delivery systems have demonstrated promise, each still has important limitations including low encapsulation efficiency, complex manufacturing, and relatively poor stability. Most of these approaches induce non-specific endosomal uptake of cGAMP-loaded particles and this forced non-specific cGAMP uptake and STING activation 
into all cell types leads to unwanted toxicity. We and others recently showed that different cell types have different cGAMP importer profiles and therefore different abilities to uptake free extracellular cGAMP. ${ }^{[25,26]}{ }^{[27]}[28]$ This cell-type specific cGAMP uptake efficiency is crucial for the potent efficacy of viral infection-induced cGAMP production at which concentrations only selective and desirable cellular cGAMP import occurs. We envision that a successful cGAMP delivery platform mimicking the natural slow release of extracellular soluble cGAMP would be highly effective, simple to manufacture at large scale, and easily loaded with diverse antigen cargo for utility across numerous disease indications.

To overcome the limitations of current subunit vaccine delivery systems while achieving slowrelease of soluble cGAMP, our lab developed an injectable polymer-nanoparticle (PNP) hydrogel that is simple to make, scalable, and readily loaded with diverse cargo. Our hydrogel vaccines act through two main mechanisms: (i) vaccine cargo are released slowly over timescales relevant to a natural viral infection and (ii) immune cells infiltrate the hydrogel to interact with high local levels of both antigen and adjuvant in a de novo immune niche. ${ }^{[29,30]}$ In this work, we evaluated whether our PNP hydrogel vaccine delivery platform could overcome the delivery challenges of cGAMP, improving cGAMP's capacity to act as an adjuvant in the context of a SARS-CoV-2 RBD subunit vaccine. We envisioned the hydrogel system could mirror biological contexts such as viral infection or cancer in which endogenous cGAMP production is localized, sustained, and acts as a powerful immune potentiator. ${ }^{[15,31]}$ Herein, we show that a subcutaneous hydrogel immunization containing cGAMP, the commonly used adjuvant Alhydrogel (alum), and RBD achieves a potent and durable humoral response in mice. As compared to dose-matched bolus controls, hydrogel vaccines led to significantly higher anti-RBD antibody titers that were maintained against recent SARS-CoV-2 variants of concern. Antibody subtype titers demonstrated that inclusion of cGAMP in the hydrogel or bolus immunizations improved skewing towards a Th1 (i.e., cell-mediated response), which is thought to be central to fighting the SARS-CoV-2 virus. ${ }^{[32]}$ Moreover, sera from hydrogel vaccinated animals possessed significantly greater neutralizing ability compared to bolus 
controls as evaluated by a pseudotyped SARS-CoV-2 infectivity assay. Finally, we demonstrated that inclusion of cGAMP as an adjuvant in the hydrogel improves recruitment of immune cells to the hydrogel vaccine niche to promote development of a rapid anti-RBD antibody response. Together, these results establish our PNP hydrogel as an effective delivery system for cGAMP in the context of an RBD-based SARS-CoV-2 subunit vaccine.

\section{Results}

\subsection{Injectable PNP hydrogels enable sustained release of cGAMP}

We have previously reported that PNP hydrogels, which are readily formed by mixing hydrophobically-modified hydroxypropylmethylcellulose (HPMC- $\mathrm{C}_{12}$ ) and biodegradable polymeric nanoparticles (NPs) made of poly(ethylene glycol)-b-poly(lactic acid), can enhance the immunogenicity and safety of model antigens and clinically de-risked adjuvants by controlling their delivery. ${ }^{[29,30,33,34]}$ Here, we employed the PNP hydrogel system to encapsulate SARS-CoV-2 RBD antigen and cGAMP as an adjuvant (Figure 1a). We also chose to include alum, which has served as a gold-standard base adjuvant in hepatitis $A$ and $\mathrm{B}$, diptheria-tetanus, and pneumococcal vaccines, and is known to adsorb negatively charged proteins and molecules to further promote controlled release. ${ }^{[35]}$ The resulting hydrogel-based vaccines are easily injected using standard syringe and needle, and self-heal following extrusion through a needle to form a solid depot in the subcutaneous space (Figure 1b). ${ }^{[3]}$ The hydrogel enables sustained release of the vaccine cargo, but also serves as an inflammatory niche into which immune cells traffic to encounter antigen and adjuvant at high local concentrations. 
a

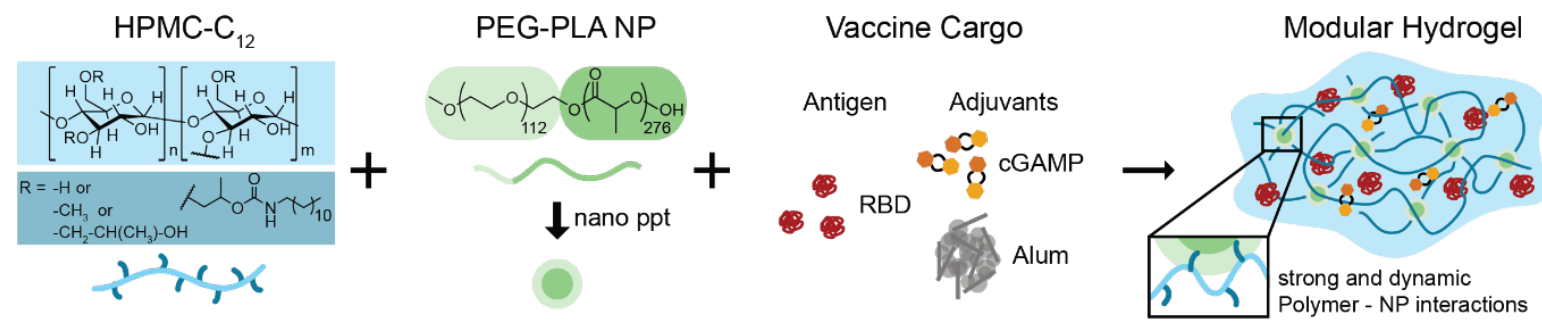

b

C
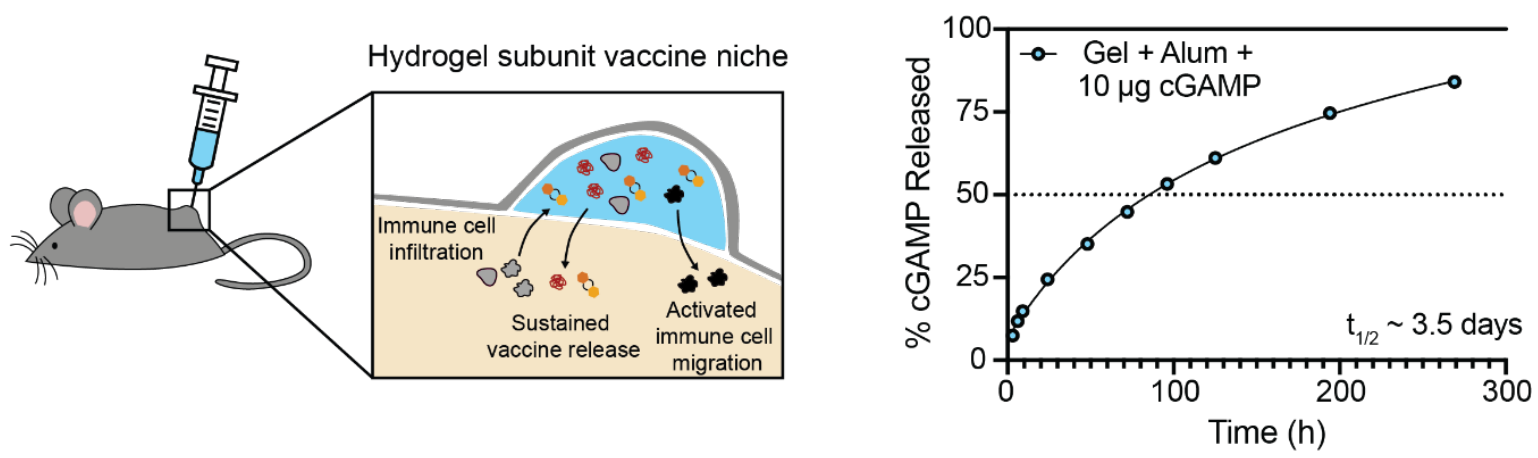

Figure 1. Polymer-nanoparticle (PNP) hydrogels provide prolonged co-delivery and generate an immune cell-activating niche for subunit RBD vaccine components. (A) Dodecyl-modified hydroxypropylmethylcellulose (HPMC- ${ }_{12}$ ) is combined by simple mixing with poly(ethylene glycol)-b-poly(lactic acid) (PEG-PLA) and vaccine cargo (RBD, cGAMP, and alum) to form PNP hydrogels. Dynamic and multivalent noncovalent interactions between the HPMC- $\mathrm{C}_{12}$ polymer and NPs leads to reversible physical crosslinking within the hydrogel. (B) Hydrogel vaccines are injected subcutaneously, forming a solid-like depot under the skin that releases vaccine components slowly over time and provides an inflammatory niche for immune cell infiltration. ${ }^{[2,5,8,29,30]}$ The RBD protein was chosen as the antigen because it is stable, easy to manufacture, and is well-conserved in emerging SARS-CoV-2 variants. ${ }^{[2,5,8]}$ (C) Percent cGAMP released over time from a hydrogel vaccine in infinite sink conditions in vitro $(\mathrm{n}=3$ from one experiment). Mean +/- SD displayed along with "One site - Total" curve fit in GraphPad Prism.

We first assessed the in vitro release kinetics of vaccine components from the hydrogel. Our prototype vaccine hydrogels were loaded into capillary tubes and incubated with saline buffer at $37^{\circ} \mathrm{C}$. The buffer sink was completely exchanged at the indicated times and the amount of vaccine component released into solution quantified in each sample. Controlled release of cGAMP from the hydrogel resulted in a release half-life of roughly 3.5 days (Figure 1c), which is similar to the half-life of RBD release in vivo previously observed $\left(t_{1 / 2}=5.5\right.$ days $) \cdot{ }^{\left[{ }^{[0]}\right.}$ Based on the diffusivity of cGAMP in PBS determined using the Stokes-Einstein equation, the halflife for cGAMP diffusion from the injection site when delivered in a PBS bolus can be estimated to be approximately 3 minutes, further supporting the hypothesis that the PNP hydrogel system 
can improve pharmacokinetics of cGAMP delivery by significantly slowing the rate of diffusion from the injection site. ${ }^{[36]}$

\section{2. cGAMP-adjuvanted hydrogels promote acute IFN signaling followed by rapid and consistent elicitation of a durable anti-SARS-CoV-2 RBD antibody response}

We next wanted to evaluate the effect of controlling cGAMP and RBD delivery from hydrogels on elicitation of humoral immunity. We therefore prepared vaccine variants in either bolus or PNP hydrogel forms that contained RBD (10 $\mu \mathrm{g})$, alum $(115 \mu \mathrm{g})$, and cGAMP (10 $\mu \mathrm{g}$ or $50 \mu \mathrm{g})$. Additionally, we sought to compare the adjuvant effect of hydrogel-delivered cGAMP versus that of poly(I:C) $(50 \mu \mathrm{g})$, a high-molecular weight polymeric toll-like receptor 3 (TLR3) and RIGI like receptor (RLR) agonist commonly employed in vaccines and evaluated in our original demonstration of PNP hydrogel-based vaccines. ${ }^{[29]}$ Vaccines were subcutaneously administered to 8-week old C57BL/6 mice at week 0 (prime) and week 8 (boost), with sera collected at weeks 1-12 (Figure 2a).

cGAMP activation of STING leads to production of type I interferons consisting of the IFN $\alpha$ cytokine family and IFN $\beta$, which trigger expression of genes that upregulate the effector function of immune cells like dendritic cells (DCs), T cells and B cells. ${ }^{[37]}$ For this reason, we assessed the acute IFN $\alpha$ response in sera 3- and 24-hours after the initial immunization (Figure S1a, b). We observed cGAMP dose-dependent IFN $\alpha$ levels in serum at 3- and 24hours and negligible levels of IFN $\alpha$ in the absence of cGAMP. The 10 and $50 \mu \mathrm{g}$ cGAMP hydrogel groups had slightly elevated levels of IFN $\alpha$ compared to the dose-matched bolus groups at the early 3-hour time point but had similar IFN $\alpha$ levels by 24 hours. Strikingly, IFN $\alpha$ levels following vaccination with poly $(\mathrm{I}: \mathrm{C})$, which is also known to be a potent type I interferon producer, were undetectable at both time points. Together, these results support the notion that cGAMP, and particularly cGAMP delivered in our hydrogel, serves as a potent IFN $\alpha$ inducing adjuvant. 
To analyze the kinetics of antibody development, we measured anti-RBD total IgG endpoint titers across eight timepoints (Figure 2a, b). With a single bolus vaccine dose, antibody titers were low or below the limit of detection for most mice, irrespective of cGAMP inclusion. In contrast, at two weeks following a control hydrogel prime dose, the average anti-RBD endpoint titer was $3.5 \times 10^{3}$, with addition of poly $(\mathrm{l}: \mathrm{C})$ increasing the response by 4.4 -fold $(P=0.34)$ and addition of $10 \mu \mathrm{g}$ or $50 \mu \mathrm{g}$ cGAMP increasing the response by 6.6 -fold $(P=0.0004)$ or 5.6 -fold $(P=0.001)$, respectively (Table S1). Notably, hydrogels including cGAMP as an adjuvant resulted in anti-RBD titers that rose most rapidly and had the smallest titer deviation across animals, both of which are desirable vaccine characteristics. The adjuvant effect of cGAMP in the hydrogel was sustained over the two months following a single vaccine dose: at weeks 4 and 8, cGAMP-hydrogel titers remained elevated, by 4.2- to 4.7-fold over the control hydrogel formulation $(10 \mu \mathrm{g}$ cGAMP hydrogel: $\mathrm{P}=0.036$ at week 4 and $\mathrm{P}=0.047$ at week $8 ; 50 \mu \mathrm{g}$ cGAMP hydrogel: $\mathrm{P}=0.046$ at week 4 and $\mathrm{P}=0.14$ at week 8; Table S1). Poly(l:C) incorporation into the hydrogel eventually led to titers 2.8-fold above hydrogel control at week $4(P=0.51)$ and 4.9-fold above hydrogel control at week $8(P=0.26)$, but the titer increase was relatively slow and more heterogeneous than was observed when cGAMP served as the hydrogel adjuvant (Table S1). Notably, prior to boosting at week 8 , the $10 \mu \mathrm{g}$ cGAMP hydrogel and $50 \mu$ g cGAMP hydrogel titers were over 200 -fold $(P<0.0001)$ and 300 -fold $(P<0.0001)$ greater than their respective bolus controls containing an equivalent cGAMP dose (Table S2). 

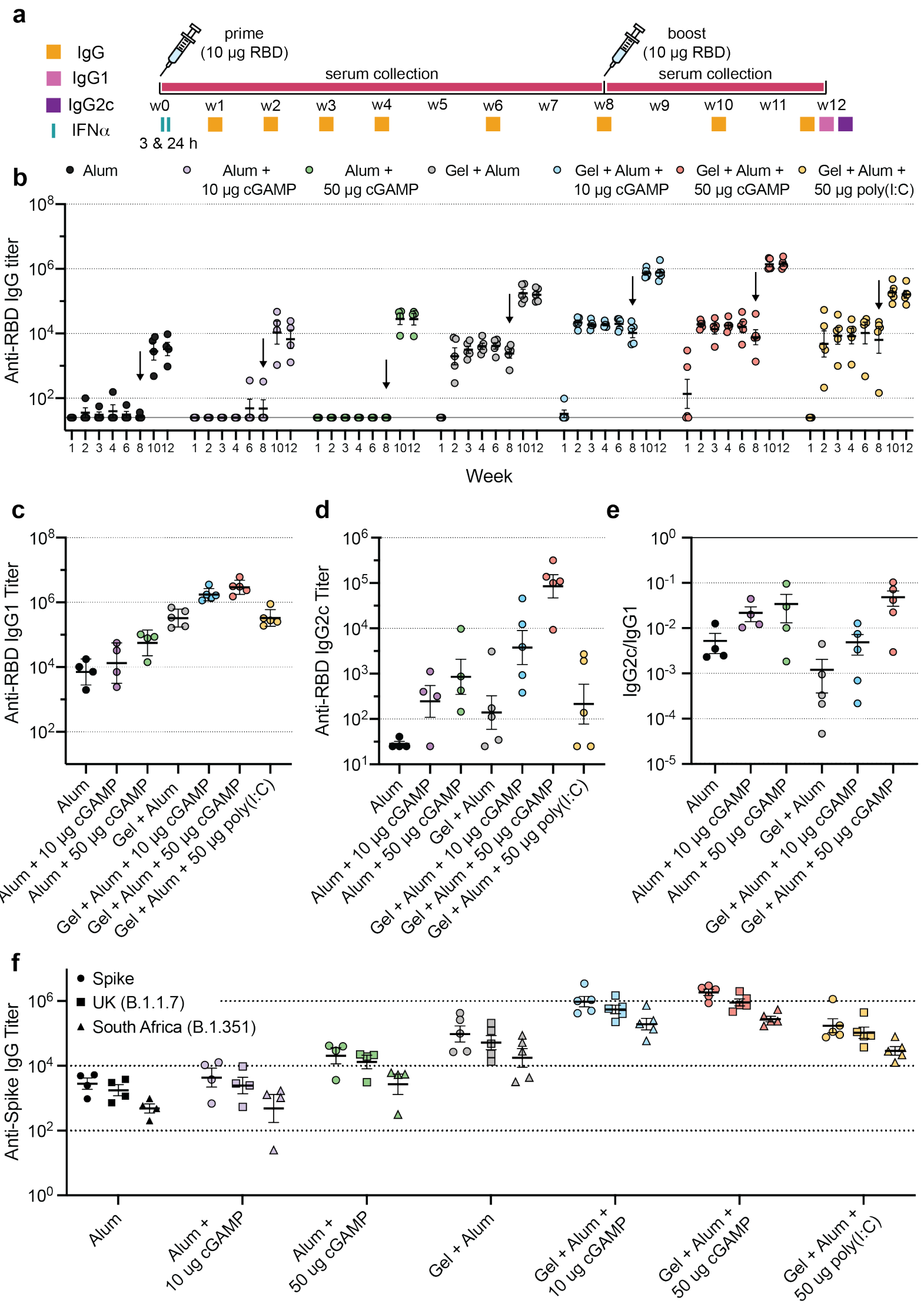

Figure 2. cGAMP-adjuvanted hydrogels elicit rapid, robust antibody responses against RBD and spike variants. (A) Vaccination schedule and timing of ELISAs. Mice were immunized at week 0 and week 8 , serum was collected periodically over 12 weeks and assays were performed as indicated. (B) Weekly anti-RBD total IgG ELISA titers before and after boosting (arrow). (C-D) Anti-RBD IgG1 (C) and IgG2c (D) titers from post-boost, week 12 serum. (E) 
Calculated ratio of anti-RBD IgG2c to IgG1 post-boost titers. Values closer to 1 suggest a balanced Th2/Th1 response whereas values well-below 1 suggest Th2 skewing towards a stronger humoral response. (F) Post-boost anti-spike IgG titers against the native spike trimer as well as the UK (B.1.1.7) and South Africa (B.1.351) variants. (B-F) Each point represents an individual mouse $(n=4-5)$. Data are shown as mean $+/$ - SEM. $P$ values listed in the text were determined using a 2way ANOVA with either Dunnett's or Tukey's multiple comparisons test (depending on number of groups compared) on the logged titer values for IgG titer comparisons (Table S1-3). P values were determined using a 2way ANOVA with Dunnett's multiple comparisons test on logged titer values for $\lg \mathrm{G} 1, \lg \mathrm{g} 2 \mathrm{c}$, and spike variant titer comparisons (Table S4-5).

Following administration of a boosting dose at week 8, anti-RBD titers were reliably detected in each bolus delivery group, with $10 \mu \mathrm{g}$ and $50 \mu \mathrm{g}$ cGAMP increasing 12-week titers above alum bolus levels by 2.5 -fold $(P=0.31)$ and 7.6 -fold $(0.0003)$, respectively (Table S3). The advantage of cGAMP adjuvanted alum hydrogels was again apparent following the boost, where incorporation of $10 \mu \mathrm{g}$ or $50 \mu \mathrm{g}$ of cGAMP increased post-boost responses by 5 -fold (P $=0.031)$ or 8.5 -fold $(P=0.0015)$, respectively, above the control hydrogel at week 12 (Table S1). In contrast, inclusion of poly $(\mathrm{I}: \mathrm{C})$ into the hydrogel provided no significant benefit at week 12. Ultimately, cGAMP hydrogel groups achieved anti-RBD titers of $8.8 \times 10^{5}$ and $1.5 \times 10^{6}$ for the 10 and $50 \mu \mathrm{g}$ cGAMP doses, respectively, which represent over 100 -fold $(P<0.0001)$ and 50 -fold $(P<0.0001)$ increases in titer for respective hydrogel groups compared to dosematched bolus administration (Table S2).

We next evaluated the isotypes of IgG that contributed to overall titers at week 12 , which serve as an indicator of how each adjuvant was influencing immune signaling. Specifically, elicitation of $\lg \mathrm{G} 1$ is associated with Th2 dominated immune responses, while $\lg \mathrm{g} 2 \mathrm{c}$ is associated with Th1-mediated skewing. ${ }^{[38]}$ Alum and the hydrogel system are independently associated with strong, Th2-skewed humoral immunity, and we observed that the major isotype elicited in all vaccine groups was indeed lgG1 (Figure 2c). ${ }^{[30,39]}$ Inclusion of $10 \mu \mathrm{g}$ or $50 \mu \mathrm{g}$ cGAMP in the hydrogel further elevated IgG1 titers by 4.9 -fold $(P=0.0006)$ or 8.5 -fold $(P<0.0001)$, respectively (Table S4). While IgG2c titers were generally lower than those of IgG1, IgG2c titers were boosted in a cGAMP dose-dependent manner. As compared to hydrogel control, adding cGAMP at $10 \mu \mathrm{g}$ or $50 \mu \mathrm{g}$ increased IgG2c titers by $\sim 20$-fold $(P=0.035)$ and $\sim 200$-fold 
$(P=0.0002)$ (Figure 2d; Table S4), therefore elevating the relative $\lg$ G2c/lgG1 ratio towards a more balanced response (Figure 2e). This observation is not surprising as numerous groups have described a role for cGAMP in balancing Th1/Th2 responses, predominantly through (i) increasing inflammatory cytokine signaling and (ii) eliciting cellular immunity which can be a crucial partner of humoral immunity in clearing certain infections. ${ }^{[24,40]}$

To confirm that the anti-RBD antibodies elicited from subunit vaccination cross-react with RBD presented in the context of native SARS-CoV-2 spike protein trimers, we then measured antispike IgG titers. Additionally, we sought to determine the degree to which antibody titers were influenced by residue substitutions in the spike protein associated with emerging SARS-CoV2 variants of concern, including that from B.1.1.7 (Alpha, UK) and B.1.351 (Beta, South Africa). Across groups, average endpoint titers against the native spike trimer and B.1.1.7 variant were largely similar, but decreased to a larger degree for the B.1.351 strain, which is understood to be an immune escape variant. ${ }^{[41]}$ The fold decrease in titer against native trimer compared to the B.1.351 variant was only $1.9(P=0.033)$ for the $10 \mu \mathrm{g}$ cGAMP hydrogel while it was $4.1(P$ $<0.0001)$ for the bolus equivalent and $11.8(\mathrm{P}=0.007)$ for the poly $(\mathrm{I}: \mathrm{C})$ hydrogel (Figure $2 \mathrm{f}$; Table S5). Notably, although titers decreased slightly against both the UK and South African variants for the 10 and $50 \mu \mathrm{g}$ cGAMP hydrogel groups (along with all other groups), titers remained well above the comparable titers for the native trimer observed for bolus vaccine controls (127- and 59-fold, respectively), demonstrating that hydrogel vaccines provided an improved humoral response compared to bolus controls, even against novel variants of concern (Figure 2f).

\section{3. cGAMP increases neutralizing antibody titers when added as an adjuvant to RBD hydrogel vaccines}

We then sought to determine if the elevated antibody titers found in GAMP-adjuvanted hydrogel groups translated to differences in neutralizing activity of the sera. We employed a reporter lentivirus pseudotyped with SARS-CoV-2 spike and measured serum-mediated 
inhibition of viral entry into HeLa cells overexpressing ACE2. ${ }^{[42,43]}$ We first surveyed 12-week sera from all vaccine conditions at a single dilution of 1:250 (Figure 3a). Sera from bolus vaccinated groups was found to have no or minimal effect on viral infectivity. More appreciable neutralizing activity was observed in sera from most animals in hydrogel vaccine control and poly $(\mathrm{l}: \mathrm{C})$ hydrogel vaccination groups, whereby infectivity decreased to $\sim 30 \%$ of total on average, although heterogeneity in the response was notable. In contrast, significant neutralizing activity was found in sera from all animals vaccinated with cGAMP adjuvanted hydrogels, with average relative infectivity reduced to just $1-3 \%$ of total.

a

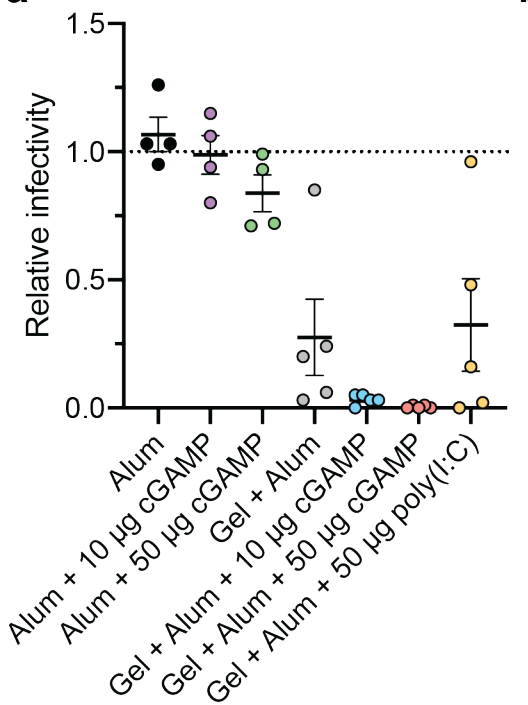

b

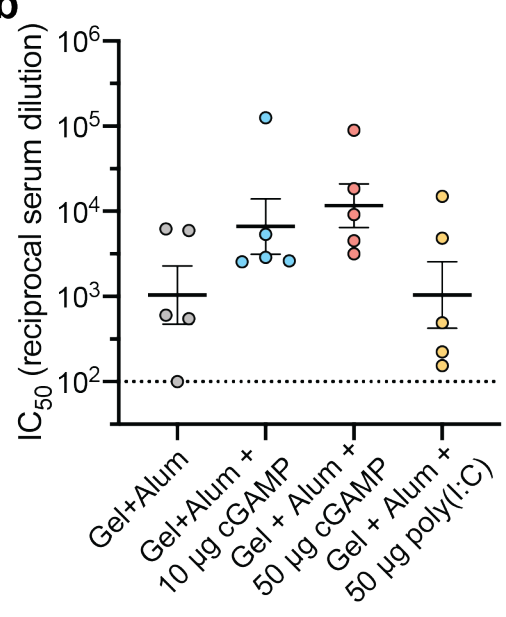

C

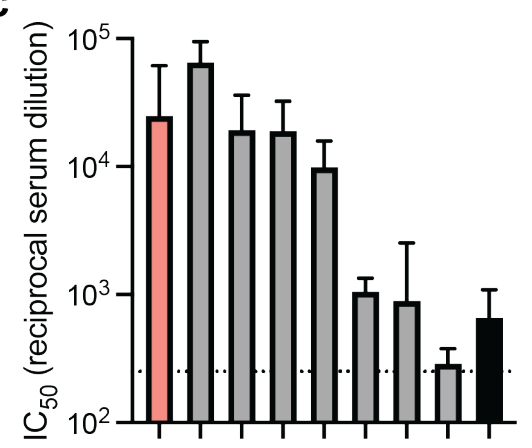

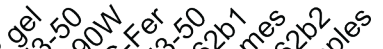

Figure 3. cGAMP-adjuvanted hydrogels provide greater neutralizing antibody titers than bolus and non-adjuvanted controls. (A) Percent infectivity across treatment groups at a 1 to 250 serum dilution. (B) $I C_{50}$ values determined from neutralization curves (D-G). Samples with minimal or undetectable neutralizing activity at the 1 to 250 dilution $(A)$ are excluded. (C) Comparison of $\mathrm{IC}_{50}$ values following immunization of mice with various $\mathrm{RBD}$ or spike constructs. Human convalescent serum data includes samples collected from patients 4-10 weeks after symptom resolution. Human data are compiled from two publications. ${ }^{[30,44]}$ Data are shown as mean + s.d. Data were extracted from published plots using the following data extraction software: https://apps.automeris.io. Dashed line represents the FDA recommendation for "high titer" classification $\left(\mathrm{IC}_{50} \sim 10^{2.4}\right) \cdot{ }^{[45]}$ Details including references, construct type, dose, immunization timeline, route of administration can be found in supplemental Table S7. (A-B) Data are shown as mean +/- SEM. $P$ values listed in the text were determined using an Ordinary one-way ANOVA with Dunnett's multiple comparisons test to compare ${ } \mathrm{C}_{50}$ values (Table S6).

We then sought to further quantify the neutralizing potency by assaying a range of sera concentrations from the hydrogel groups in which we observed significant inhibition of infectivity. We determined that the half maximal inhibition of infectivity $\left(\mathrm{IC}_{50}\right)$ for control- and 
poly(I:C)-hydrogel groups occurred at reciprocal dilutions of $2.7 \times 10^{3}$ and $4.1 \times 10^{3}$, respectively

(Figure 3b, Figure S2a-d). In comparison, sera from hydrogel groups adjuvanted with $10 \mu \mathrm{g}$ or $50 \mu \mathrm{g}$ cGAMP had $\mathrm{IC}_{50}$ values of $2.8 \times 10^{4}(\mathrm{P}=0.24$ compared to Gel+Alum control) and $2.5 \times 10^{4}(P=0.098$ compared to Gel+Alum control) reciprocal dilutions, respectively, corresponding to an order of magnitude increase in neutralizing activity relative to the control hydrogel group (Table S6). Taken together, these data demonstrate that (i) PNP hydrogelbased vaccine administration provides significant benefit for elicitation of humoral immunity versus bolus administration and (ii) adjuvanting hydrogels with cGAMP further boosts elicitation of functional, protective antibodies. We also compared our neutralization results to other COVID-19 mouse immunization studies by plotting a set of $\mathrm{IC}_{50}$ values found in the literature (Figure 3c, Table S7). Our 50 mg cGAMP hydrogel containing wildtype RBD resulted in $\mathrm{IC}_{50}$ values well over $10^{4}$, greatly exceeding the FDA "high titer" cutoff of $1 \mathrm{C}_{50} \sim 10^{2.4}$. ${ }^{[45]}$ Notably, the $50 \mu \mathrm{g}$ cGAMP hydrogel $\mathrm{IC}_{50}$ value is comparable to other leading subunit vaccine candidates containing RBD or Spike constructs found in the literature (Figure 3c, Table S7). ${ }^{[44 \text {, }}$ 46, 47] These results provide proof of principle that controlled release of cGAMP from hydrogelbased vaccines represents an effective strategy to unleash the adjuvant activity of a molecule with a notoriously challenging pharmacokinetic profile.

\section{4. cGAMP promotes migration of numerous immune cell types to the hydrogel vaccine} niche

We previously found that PNP hydrogels created a physical inflammatory niche into which cells can infiltrate. ${ }^{[29]}$ Moreover, the antigen and adjuvant cargo crucially shape the nature of the inflammatory microenvironment formed within the gel. We therefore evaluated how addition of cGAMP influences the degree and composition of cellular infiltrate into the hydrogel. Four days following in vivo subcutaneous injection, hydrogels were excised and then the total cellular infiltration was quantified via flow cytometry (Figure 4a). While control hydrogels contained $5.3 \times 10^{5}$ live cells on average, addition of cGAMP increased the average infiltrating cell number in the gel to $1.1 \times 10^{6}$ cells (Figure $4 b$ ), with some heterogeneity in the magnitude of the effect 
observed across animals. Of the cells infiltrating the vaccine hydrogel, the vast majority were CD45+ leukocytes (Figure 4c).

Addition of cGAMP to hydrogels resulted in higher average counts of several important immune subsets compared to control hydrogels containing alum and RBD alone, including NK cells (Figure 4d) and most notably both $\mathrm{T}$ helper cells (CD4 ${ }^{+}$Th-cells, Figure $\left.4 \mathrm{f}\right)$ and cytotoxic $\mathrm{T}$ lymphocytes $\left(\mathrm{CD}^{+} \mathrm{CTL}\right.$, Figure $\left.4 \mathrm{~g}\right)$ as well as conventional dendritic cells type 2 (cDC2, Figure 4j). Additional subsets which showed non-significant changes in gel infiltration were NKT cells (Figure 4e), myeloid cells (Figure 4h), and conventional dendritic cells type 1 (cDC1, Figure 4i). In examining how the percent representation of each subset shifted among the total number of infiltrating cells (Figure 4I), the most pronounced effect of cGAMP appeared to be

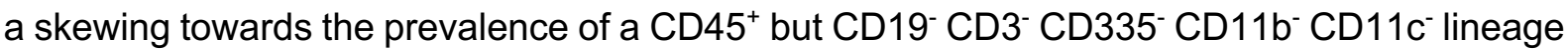
negative subset (Figure 4k), which we hypothesize may represent innate lymphoid cells (ILCs), although the addition of further surface markers to the flow cytometry panel will be necessary to confirm the ILC identity. Although counts were low at this time point, we observed an increase in both $\mathrm{CD} 4^{+}$and $\mathrm{CD} 8^{+} \mathrm{T}$ cells in the presence of cGAMP (Figure $4 \mathrm{f}-\mathrm{g}$ ). The ratio of $\mathrm{CD}^{+} / \mathrm{CD}^{+} \mathrm{T}$ cells for the cGAMP hydrogel group was about 1.9 suggesting there is slight skewing towards T helper cells over cytotoxic T cells. These results suggest that cGAMP plays an early and appreciable role in affecting cellular migration to the site of the vaccine depot, which we predict contributes to downstream benefits in antigen presentation and cellular activation that enable the enhanced humoral responses we observed in these vaccine groups. 
a
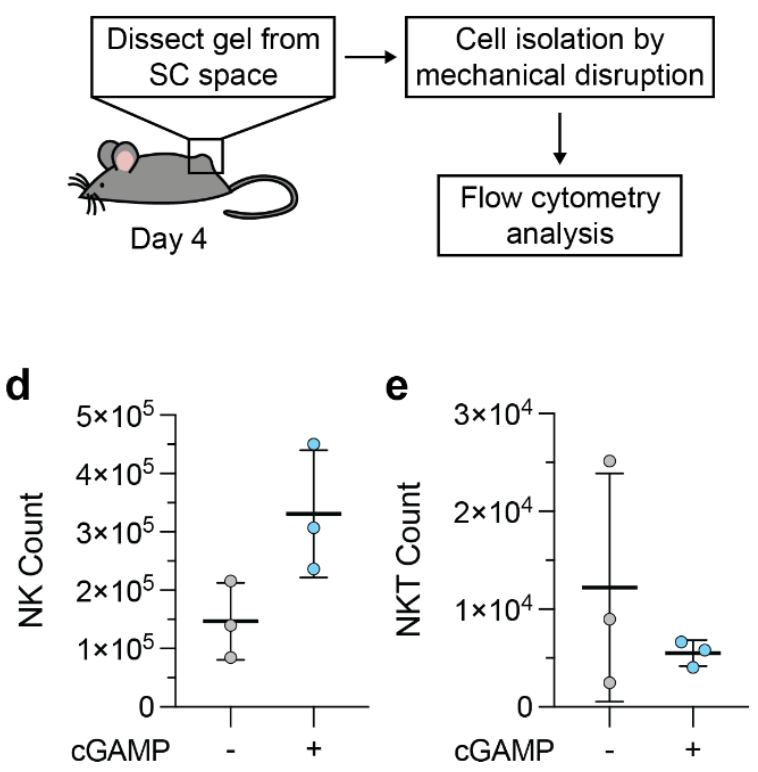

h
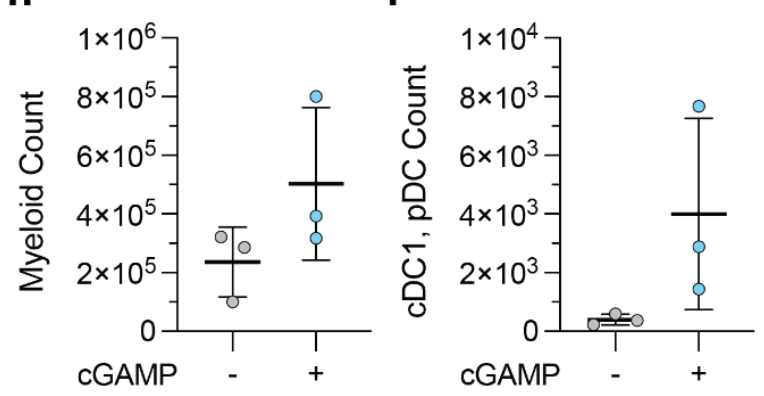

\section{b}

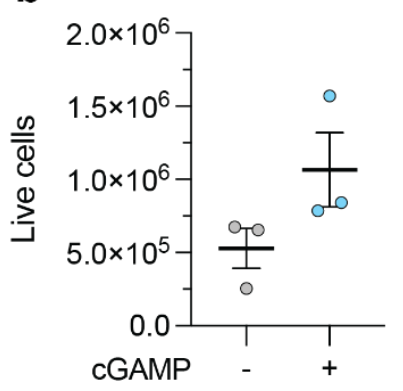

f

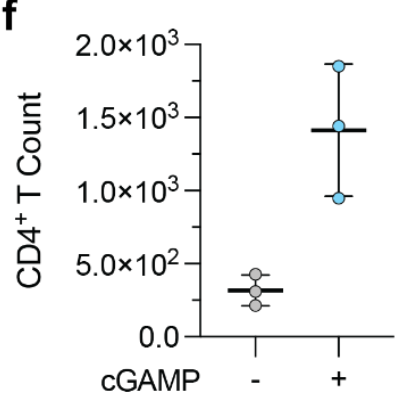

j

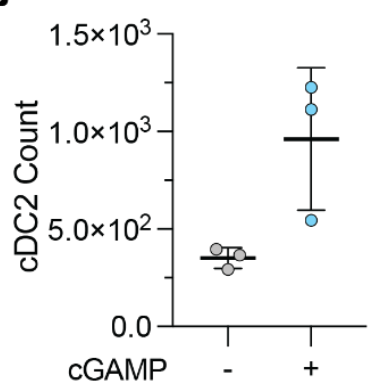

C
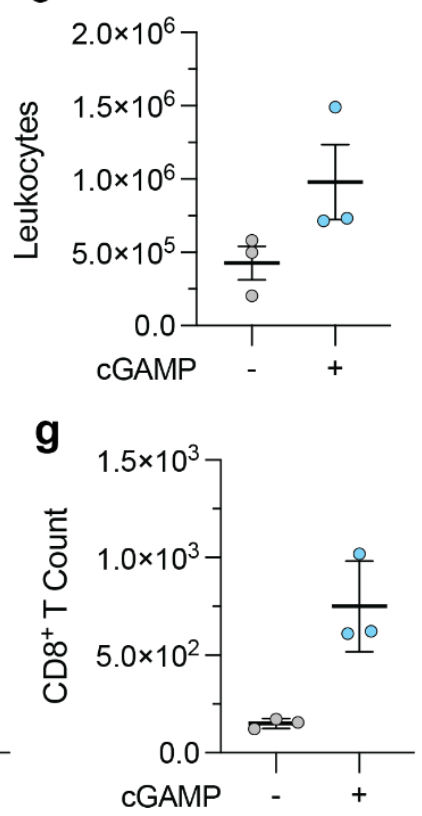

k

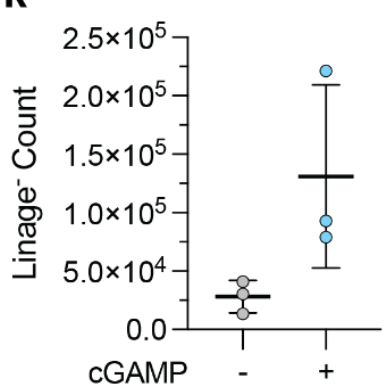

I
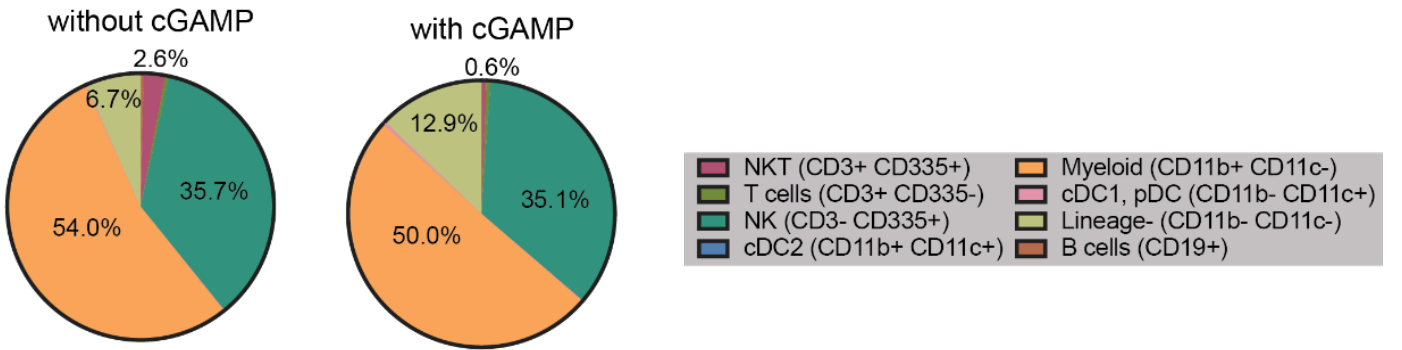

Figure 4. Characterization of cellular infiltrate to the hydrogel vaccine niche. (A) Schematic of the experimental workflow: four days following injection of hydrogels containing RBD, alum, and the absence or presence of $10 \mu \mathrm{g}$ cGAMP, gels were excised and dissociated. Total cellular infiltrate was then analyzed by flow cytometer. (B-K) Flow cytometry counts of live cells, $\mathrm{CD} 45^{+}$leukocytes, and CD45 ${ }^{+}$immune subsets. (I) Percentages of major immune subsets among total $\mathrm{CD}_{4} 5^{+}$infiltrate, without or with $10 \mu \mathrm{g}$ cGAMP. For $(\mathrm{B}-\mathrm{K}), \mathrm{n}=3$ mice with error bars representing mean \pm s.d.; $p$ values are determined by a two-tailed $t$ test. For $(I)$, the average percentage across $n=3$ mice is displayed. 


\section{5 cGAMP leads to systemic shifts in immune cell populations and both activation and antigen uptake in antigen-presenting cells in the gel}

Since cGAMP elicits such a strong humoral response leading to superior antibody titers and neutralization properties, we reasoned that it must act early on antigen-presenting cells (APCs), leading to efficient activation of these cells as well as antigen transport and subsequent presentation to adaptive immune cells like $T$ cells and B cells. Upon entry into the responder cells, cGAMP binds to the adaptor STING, leading to STING phosphorylation and the subsequent phosphorylation and activation of its downstream transcription factor IRF3. To determine the direct effects of cGAMP on APCs, we stained for phosphorylated STING (pSTING) and phosphorylated IRF3 (pIRF3), which is downstream of STING activation but can also be an indirect activation signal through type I IFN receptor signaling. To assess the uptake of antigen into APCs, we used an anti-His antibody to stain for the His-tagged RBD. With these experimental parameters in place, we compared immune cell subsets found in implanted hydrogels, the draining inguinal lymph node, and the spleen following the same experimental setup as laid out in Figure 4a.

One particular cell type of interest in these studies was DCs, which represent an important link between innate and adaptive immunity. As professional APCs, DCs sample both soluble and particulate antigen and, when activated by the right accompanying signals, present these antigens to activate T cell responses. ${ }^{[48]}{ }^{[49]}$ Different DC subsets show distinct functions in response to an infection. Inflammatory DCs (iDCs) are thought to be derived from monocytes and have been shown to efficiently shuttle antigen from the site of infection to the draining lymph node to elicit a T cell response. ${ }^{[50]}$ Conventional dendritic cells (cDCs) are sub-divided into $\mathrm{CDC} 1$ and $\mathrm{cDC} 2 \mathrm{~s}$. $\mathrm{cDC} 1 \mathrm{~s}$ cross-present exogenous antigen on $\mathrm{MHC}$ I for activation of cytotoxic T lymphocytes (CTL), which are instructed to kill infected cells. cDC2s, while also able to cross-present, present their antigen on MHC II molecules and are thus the main activators of helper T cells (Th cells), which in turn are crucial for activation of both CTL and the humoral response mediated by antibody-producing B cells. ${ }^{[11,19]}$ 
We encountered an elevated RBD signal in monocytes (Figure 5a) and iDCs (Figure 5b) with cGAMP incorporation in hydrogels, indicating that these cell types efficiently take up antigen when cGAMP is present. Interestingly, iDCs in the hydrogel were also pSTING positive, suggesting that they are directly activated by cGAMP (Figure S4a), although there was no significant difference in pIRF3 in these cells. In contrast, monocytes were pSTING negative but pIRF3 positive, suggesting these cells either directly respond to cGAMP, but pSTING activation is too transient, or they are indirectly activated by type I IFN. We observed an increase of iDCs only in the lymph node (Figure S4a), which is not surprising as inflammatory DCs migrate from the site of infection to the draining lymph node. ${ }^{[50]}$ Of note, monocytes and iDCs were RBD positive exclusively in the hydrogel niche, whereas the pIRF3 signal of monocytes was also detectable in the lymph node, with none of the signals observable in the spleen, highlighting the superior pharmacodynamics of our hydrogel-cGAMP-RBD system in activating iDCs.

Next, we evaluated cDC subsets. Since we observed high IgG1 titers as well as IgG2c titers (Figure 2c-e) and found significantly more infiltration of cDC2 but not cDC1 cells into the hydrogel when cGAMP is present (Figure $4 \mathrm{i}, \mathrm{j}$ ), we hypothesized that we may detect differences in $\mathrm{cDC} 1 / \mathrm{cDC} 2$ activation as well. Indeed, the majority of cDCs found in the hydrogel depot were $c D C 2 s$, with the lymphoid tissue-specific $C D 8^{+} \mathrm{CDC} 1$ population virtually non-existent in the hydrogel, which is also reflected by a lack of CD8 $\alpha$ expression on these cells (Figure 5c and Figure S4b). In both the lymph node and the spleen, cDC1s decreased, while $c D C 2 s$ increased in the lymph node in the presense of cGAMP (Figure $5 c$ ). These findings corroborate the observation that in $\mathrm{cDCs}$, the overall expression level of CD8 $\alpha$ decreased in both lymph node and spleen, while CD11b increased in the hydrogel and lymph node (Figure S4b). In addition to a shift in $\mathrm{CDC} 1$ to $\mathrm{CDC} 2$ populations, we observed a pIRF3 signal in $\mathrm{cDC} 2 \mathrm{~s}$ residing in both the hydrogel depot and the spleen (Figure 5d), demonstrating that cGAMP leads to activation of these crucial APCs within the local inflammatory niche within the hydrogel and also systemically. 

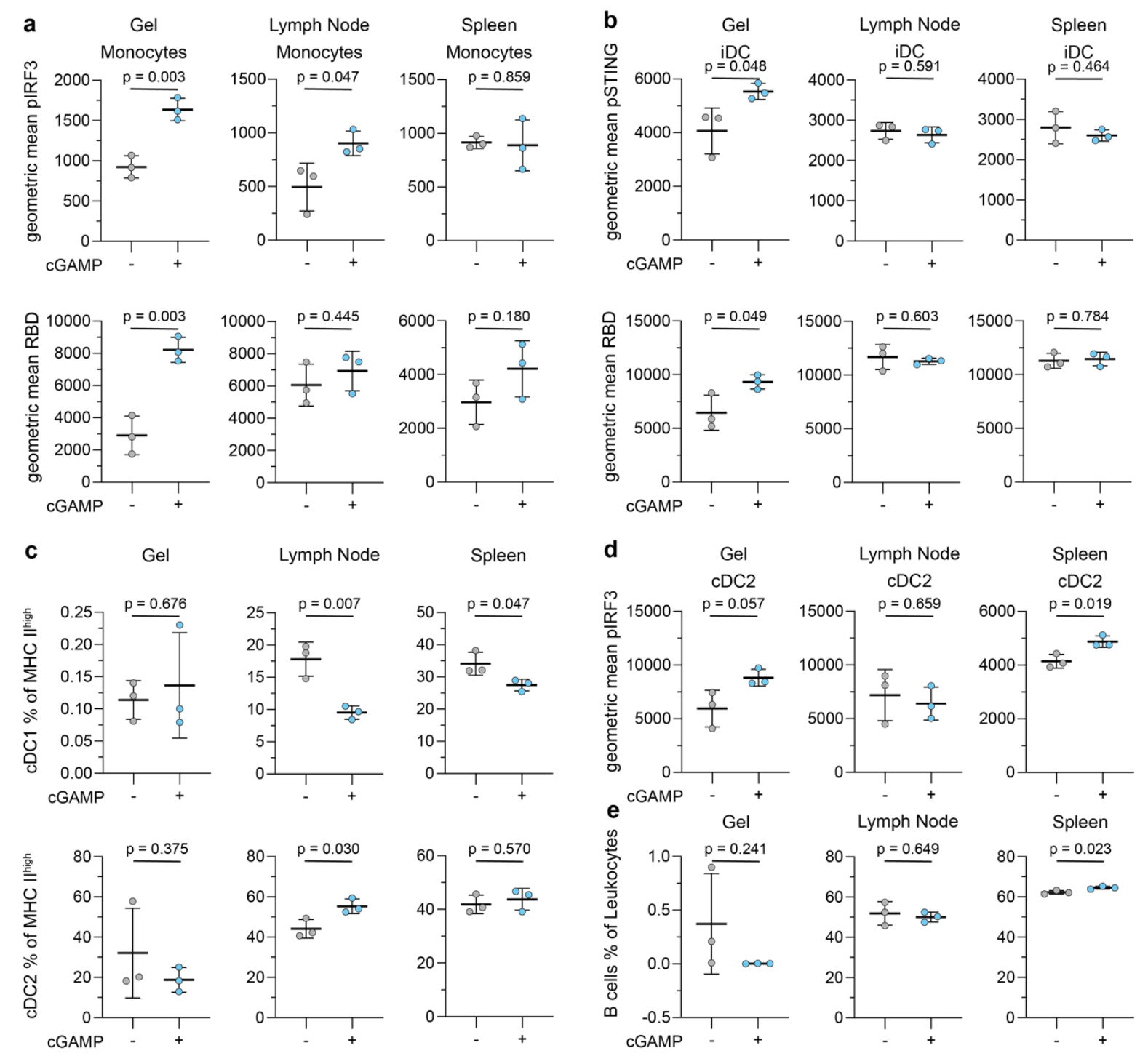

Figure 5. Further characterization of APC infiltrate to the hydrogel vaccine niche. Experiments were conducted as shown in Figure 4a. (A) geometric means of pIRF3 (upper panels) and RBD (lower panels) of monocytes (CD45 ${ }^{+}$CD 19- CD3 ${ }^{-}$CD335 CD11 ${ }^{+}$CD11 ${ }^{-}$Ly6 $^{\text {high }}$ ) in gel, draining inguinal lymph node, and spleen. (B) Geometric means of pSTING (upper panels) and RBD (lower panels) of inflammatory DCs (iDC, CD45 ${ }^{+}$CD19- CD3- CD335- $\mathrm{MHC} \mathrm{I}^{+} \mathrm{CD}^{-} 11 \mathrm{~b}^{+}$ Ly6C $^{+}$). (C) CDC1 (upper panel, CD45 ${ }^{+}$CD19- CD3 $3^{-}$CD335- CD11 ${ }^{+} \mathrm{MHC}^{-} \mathrm{II}^{\text {high }} \mathrm{F} 4 / 80^{-} \mathrm{Ly}^{-} \mathrm{C}^{-}$

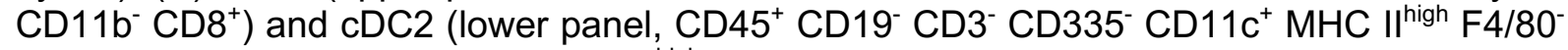
Ly6C- $\mathrm{CD}^{-} 11 \mathrm{~b}^{+} \mathrm{CD} 8^{-}$) percent of MHC II ${ }^{\text {high }}$ APCs. (D) Geometric means of pIRF3 in cDC2s. (E)

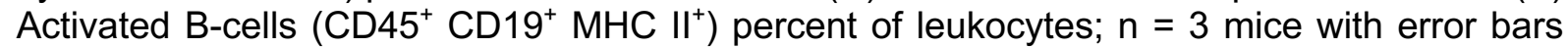
representing mean \pm s.d.; $p$ values are determined by a two-tailed $t$ test.

Even though four days after hydrogel vaccine administration is an early time point to evaluate adaptive immune cells, we attempted to gain insight into $T$ and $B$ cell dynamics. While CTLs seemed to non-significantly increase overall in the hydrogel depot, lymph node, and spleen, we observed a significant increase in Th cells in the hydrogel, while the percentage in both lymph node and spleen decreased, suggesting a shift of Th cells from both spleen and lymph node to the hydrogel niche (Figure S4c). While B cells minimally infiltrated hydrogel (Figure 
4I), we observed a significant increase in activated $\mathrm{MHC} \mathrm{II}^{+} \mathrm{B}$ cells in the spleen (Figure 5e).

These results suggest profound local and systemic shifts of immune cell populations.

Overall, we found that monocytes and iDCs predominantly took up antigen in the hydrogel depot, with both cell populations being activated by cGAMP. Moreover, cDC2s increased overall and showed a pIRF3 signal, suggesting that they were activated by the cGAMP provided in the hydrogel, ultimately leading to changes in both Th cells and activated B cells. Taken together, our observations depict the mechanisms necessary for a robust and strong cGAMP-mediated antibody response on a cellular level.

\section{Discussion}

The efficacy of subunit vaccines is determined by the selection of antigen, adjuvant(s), and the spatiotemporal control of their exposure to the immune system, all of which crucially interact to shape the resulting immune response. Both the RBD as an antigen and cGAMP as an adjuvant offer promising advantages, but each also possesses significant limitations related to ineffective delivery and poor localization within immune compartments. Here, we demonstrated that an injectable, self-assembled PNP hydrogel system simultaneously helps overcome delivery challenges posed by RBD and cGAMP, and that co-administration of these agents in this easily produced vaccine format leads to significant and functionally important increases in immunogenicity.

While the importance of targeting the RBD is illustrated by the high fraction of neutralizing antibodies that target the RBD, we and others have now shown that engineering of delivery systems for the protein domain or its multimerization are required to generate appreciable antiRBD antibody responses in vaccination. ${ }^{[8,30,51]}$ For example, we have shown previously that delivery of subunit vaccines from a PNP hydrogel depot improves the humoral response compared to a dose-matched bolus control likely because prolonged antigen release better mimics the kinetics of antigen exposure occurring during a natural infection. ${ }^{[29,30]} \mathrm{A}$ brief 
literature search showed that $\mathrm{IC}_{50}$ values from neutralization studies following immunization with our cGAMP hydrogel vaccine greatly exceeded the FDA "high titer" mark and exceeded $I_{50}$ values for both Spike- and RBD-encoding mRNA vaccines in mice. It is important to note that there were differences in dosing, timelines, and neutralization assay procedures across these studies and that $\mathrm{IC}_{50}$ values are an indication of the humoral response and not the cellmediated response, which is known to also play a role in protection from SARS-CoV-2.

A main finding from this work is that our PNP hydrogel is a suitable delivery system for cGAMP as a subunit vaccine adjuvant. Within the context of a COVID-19 vaccine, cGAMP is particularly useful as an adjuvant because of its role in inducing potent cell-mediated (Th1) responses. Previous studies have shown that less severe cases of SARS-CoV-1 were associated with rapid onset of a Th1 response, while Th2 responses were linked to greater lung inflammation and worse outcomes. ${ }^{[52]}$ Although the PNP hydrogel itself was previously found to skew towards a humoral (Th2) response, we observe here that addition of cGAMP inverted this effect in a dose-dependent manner. ${ }^{[29,30]}$ Notably, the average IgG2c/lgG1 ratio for the $50 \mu \mathrm{g}$ cGAMP hydrogel group was higher than that of the dose-matched bolus group, suggesting that hydrogel was potentiating cGAMP's ability to skew towards Th1. Future studies will reveal the degree to which cGAMP-embedded gels affect Th1 skewing at the level of cellular function, including induction of antigen-specific cytotoxic $\mathrm{CD} 8^{+} \mathrm{T}$ cells.

Another striking finding was that cGAMP clearly outperformed poly $(I: C)$ as an adjuvant within the hydrogel depot, with poly $(\mathrm{I}: \mathrm{C})$ providing no additional benefit after the boosting vaccine dose versus control gel. We speculate that several factors enable cGAMP to more rapidly, consistently, and robustly adjuvant the hydrogel-based vaccine versus poly(l:C). As a small molecule, cGAMP is more likely to be evenly distributed throughout the assembled matrix and diffuse more quickly within and out of the gel. While extracellular cGAMP must enter cells to activate STING in the cytosol, we and others recently established that cells possess specific importers that allow for the small molecule's uptake. ${ }^{[25-28]}$ In contrast, poly(l:C) is very large (1.5 
$-8 \mathrm{~kb})$ and is imported through endosomal uptake. Poly $(\mathrm{l}: \mathrm{C})$ then can activate toll-like receptor 3 within endosomes or can escape to the cytosol to activate RIG-I like receptors. ${ }^{[53]}$ The PNP hydrogel system was designed so that diverse antigen cargo can be easily loaded into it, and our results suggest that incorporation of cGAMP into subunit vaccine hydrogels of many different antigens could represent a flexible strategy for production of vaccines against other viral and infectious agents, especially those that require more durable immunity with balanced Th1/Th2 responses for clearance.

We have previously shown that the hydrogel delivery system provides sustained release of both antigens and adjuvants in vivo. ${ }^{[29,30]}$ Ideally, we would have liked to perform thorough cGAMP biodistribution analyses here to verify that the cGAMP hydrogel groups achieved high titers and neutralization through this same mechanism; however, two properties of cGAMP make these studies challenging. Since cGAMP is a small cyclic dinucleotide, it is difficult to label for tracking studies while maintaining native-like properties and activity. Moreover, cGAMP is rapidly degraded in circulation by ENPP1, which precludes direct detection of systemic cGAMP levels unless ENPP1 inhibitors are continually dosed at elevated concentrations. ${ }^{[12][13]}$ While we do not present in vivo release or biodistribution data for cGAMP, in previous work we showed that RBD was released from the hydrogel in vivo with a half-life of $\sim 5.5$ days, which is within a similar window to the half-life of $\sim 3.5$ days for cGAMP release observed here in our in vitro study. ${ }^{[30]}$

Our initial examination of the hydrogel infiltrate revealed that cGAMP is playing a major role in promoting immune cell recruitment to the inflammatory niche at the site of vaccination. The effect of cGAMP on increasing immune infiltration to hydrogels containing alum and RBD was significantly more profound than the role played by alum and RBD compared to empty hydrogels. These results suggest that cGAMP plays an early and crucial role in immune activation, as would be desired of an effective adjuvant. Intriguingly, we showed that monocytes and inflammatory DCs are activated by cGAMP and take up RBD antigen in the 
hydrogel niche four days after gel injection. These results, seen in combination with the observed systemic shift towards $\mathrm{cDC} 2 \mathrm{~s}$, activation of $\mathrm{cDC} 2 \mathrm{~s}$ in the hydrogel and apparent migration of Th cells to the hydrogel, give us initial insight as to how cGAMP as an adjuvant may work on a cellular and systemic level. While we can only speculate on migration of immune cells in this scenario, it is intriguing that iDCs seem to accumulate in the draining lymph node, while activated B cells are elevated in the spleen. Future, more in-depth spatiotemporal studies may provide a comprehensive picture, tracking cells in their migration between the gel depot and lymphoid organs. While the data suggests activation of iDCs and monocytes directly in the hydrogel depot by cGAMP, it is worth noting that these data at present do not reveal whether cGAMP is preferentially interacting with cells localized around the hydrogel, or if cGAMP that reaches the draining lymph node serves to mobilize cells to traffic to the hydrogel. It is however, likely that cGAMP only acts over short distances on account of rapid degradation by ENPP1 in the surrounding tissues. ${ }^{[15][12]}$ Future detailed biological studies are expected to shed light on the central mechanisms by which hydrogel-controlled cGAMP delivery affects innate immune cell activation state, presentation of co-delivered antigen, and the development of adaptive cellular effectors for both humoral and cell-mediated protection. While the hydrogelbased vaccines elicited antisera with appreciable in vitro neutralizing activity, assessing the degree of induced protection in vivo through viral challenge studies remains an important aspect for future work to develop cGAMP-adjuvanted subunit hydrogel vaccines for SARSCoV-2 and beyond.

\section{Conclusions}

In total, our data demonstrate that delivery of cGAMP from a PNP hydrogel containing the commonly used adjuvant, alum, and the critical SARS-CoV-2 antigen, RBD, results in significantly higher anti-RBD antibody titers and improved neutralization as compared to both dose-matched bolus controls and the hydrogel vaccine lacking cGAMP. Immunization with the cGAMP hydrogel vaccines containing RBD resulted in higher cross-reactive antibody titers against the recent SARS-CoV-2 spike protein variants that have emerged in South Africa and 
the UK. As an early indication of cGAMP's ability to act as an effective adjuvant in this system, we found that inclusion of cGAMP in the hydrogel increases infiltration of key immune populations into the hydrogel vaccine niche as early as 4 days following injection. These results support the use of PNP hydrogels as a means to overcome the delivery limitations of cGAMP and illustrate that cGAMP, when delivered in this manner, is an effective adjuvant for improving immunogenicity against SARS-CoV-2 RBD antigen. Given the findings presented here, cGAMP-adjuvanted hydrogels represent an effective and scalable subunit vaccine platform that could be readily adapted and evaluated for use against a broad range of infectious agents.

\section{Experimental Section}

\subsection{Materials}

Mammalian Cell culture: Expi293F cells (ThermoFisher) were maintained in a 2:1 mix of FreeStyle293:Expi293 Expression medium (ThermoFisher) and grown at $37{ }^{\circ} \mathrm{C}$ and $8 \% \mathrm{CO}_{2}$ while shaking at $120 \mathrm{rpm}$. HEK293T cells (ATCC) were maintained in DMEM (Cellgro) supplemented with 10\% FBS, L-glutamate, 1\% penicillin-streptomycin, and $10 \mathrm{mM}$ HEPES. ACE2/HeLa cells were generously provided by Dennis Burton and were maintained in DMEM (Cellgro) supplemented with 10\% FBS, L-glutamate, 1\% penicillin-streptomycin, and $10 \mathrm{mM}$ HEPES. ${ }^{[43]}$

Chemicals: HPMC (meets USP testing specifications), N,N-diisopropylethylamine (Hunig's base), hexanes, diethyl ether, N-methyl-2-pyrrolidone (NMP), dichloromethane (DCM), lactide (LA), 1-dodecylisocynate, and diazobicylcoundecene (DBU) were purchased from SigmaAldrich and used as received. Monomethoxy-PEG (5 kDa) was purchased from Sigma-Aldrich and was purified by azeotropic distillation with toluene prior to use. For in vitro studies, 2'3'cyclic-GMP-AMP (cGAMP) was synthesized and purified in-house as previously described. ${ }^{[25]}$ For in vivo studies, vaccine-grade 2'3'-cyclic-GMP-AMP (cGAMP) was purchased from Invivogen. 
Plasmids: The SARS-CoV-2 RBD DNA construct was kindly provided by Dr. Florian Krammer. ${ }^{[54]}$ The expression plasmid (pCAGGS) contains a CMV promoter, followed by the native SARS-CoV-2 Spike signal peptide (residues 1-14), RBD-encoding residues 319-541 from the SARS-CoV-2 Wuhan-Hu-1 genome sequence (GenBank MN908947.3), and a Cterminal hexa-histidine tag. A five-plasmid system was used for production of SARS-CoV-2 spike pseudotyped lentivirus: the lentiviral packaging vector (pHAGE_Luc2_IRES_ZsGreen), the SARS-CoV-2 spike (sequence from Wuhan-Hu-1 strain of SARS-CoV-2, GenBank NC_045512), and lentiviral helper plasmids (HDM-Hgpm2, HDM-Tat1b, and pRCCMV_Rev1b). ${ }^{[42]}$

\subsection{Methods}

Expression and purification of SARS-CoV-2 Spike RBD: The mammalian expression plasmid for RBD production was a kind gift from Dr. Florian Krammer and was previously described in detail. ${ }^{[44]}$ Transient transfection of Expi293F cells was performed at a density of $\sim 3-4 \times 10^{6}$ cells $\mathrm{mL}^{-1}$. Per liter of transfected cells, $568 \mu \mathrm{g}$ DNA in $113 \mathrm{~mL}$ culture medium was complexed with $1.48 \mathrm{~mL}$ FectoPro (Polyplus) for $10 \mathrm{~min}$ at RT. The transfection mixture was then added to cells along with boosting D-glucose (4 $\mathrm{g} \mathrm{L}^{-1}$ final) and 2-propylpentanoic (valproic) acid (3 mM final). Culture supernatants were harvested 3-5 days post-transfection via centrifugation at $7,000 \times \mathrm{g}$ for 15 minutes followed by $0.22 \mu \mathrm{m}$ filtration. Samples were snap frozen and stored at $-20^{\circ} \mathrm{C}$ until use.

HisPur NiNTA resin (ThermoFisher) was washed $3 x$ with $\sim 10$ column volumes wash buffer (10 mM imidazole in $1 \mathrm{X}$ PBS, $\mathrm{pH} 7.4)$. Culture supernatant was diluted 1:1 (10 mM imidazole in $1 \mathrm{x}$ PBS, $\mathrm{pH} 7.4$ ) and incubated with resin at $4{ }^{\circ} \mathrm{C}$ with gentle stirring. Resin/supernatant mixture was then loaded into gravity flow columns and washed $1 \mathrm{x}$ with wash buffer. Protein was eluted with $250 \mathrm{mM}$ imidazole in 1x PBS and spin concentrated (10 kDa MWCO, Amicon). Size-exclusion chromatography was performed using a GE Superdex 200 Increase 10/300 GL column pre-equilibrated in 1x Dulbecco's phosphate-buffered saline (Gibco). Fractions were evaluated by SDS-PAGE (4-20\% Mini-PROTEAN TGX, 
ThermoFisher), pooled, and spin concentrated. Purified RBD was supplemented with $10 \%$ glycerol, filtered through a $0.22 \mu \mathrm{m}$ filter, snap frozen, and stored at $-20{ }^{\circ} \mathrm{C}$.

Preparation of HPMC $-\mathrm{C}_{12}$ : $\mathrm{HPMC}-\mathrm{C}_{12}$ was prepared according to previously reported procedures. ${ }^{[29,55]} \mathrm{HPMC}(1.0 \mathrm{~g})$ was dissolved in NMP $(40 \mathrm{~mL})$ by stirring at $80^{\circ} \mathrm{C}$ for $1 \mathrm{~h}$. Once the solution cooled to RT, 1-dodecylisocynate (105 mg, $0.5 \mathrm{mmol}$ ) and $\mathrm{N}, \mathrm{N}-$ diisopropylethylamine (catalyst, $\sim 3$ drops) were dissolved in NMP (5.0 mL). This solution was added dropwise to the reaction mixture, which was then stirred at RT for $16 \mathrm{~h}$. This solution was then precipitated from acetone, decanted, re-dissolved in water ( 2 wt \%), and placed in a dialysis tube for dialysis for 3-4 days. The polymer was lyophilized and reconstituted to a 60 $\mathrm{mg} \mathrm{mL}^{-1}$ solution with sterile PBS.

Preparation of PEG-PLA NPs: PEG-PLA was prepared as previously reported. ${ }^{[29,55]}$ Monomethoxy-PEG (5 kDa; $0.25 \mathrm{~g}, 4.1 \mathrm{mmol})$ and DBU (15 $\mu \mathrm{L}, 0.1 \mathrm{mmol} ; 1.4 \mathrm{~mol} \%$ relative to LA) were dissolved in anhydrous dichloromethane $(1.0 \mathrm{~mL})$. LA $(1.0 \mathrm{~g}, 6.9 \mathrm{mmol})$ was dissolved in anhydrous DCM $(3.0 \mathrm{~mL})$ with mild heating. The LA solution was added rapidly to the PEG/DBU solution and was allowed to stir for $10 \mathrm{~min}$. The reaction mixture was quenched and precipitated by 1:1 hexane and ethyl ether solution. The synthesized PEG-PLA was collected and dried under vacuum. Gel permeation chromatography (GPC) was used to verify that the molecular weight and dispersity of polymers meet our quality control (QC) parameters. NPs were prepared as previously reported. ${ }^{[29,55]}$ A $1 \mathrm{~mL}$ solution of PEG-PLA in DMSO (50 $\mathrm{mg} \mathrm{mL}^{-1}$ ) was added dropwise to $10 \mathrm{~mL}$ of water at RT under a high stir rate (600 rpm). NPs were purified by centrifugation over a filter (molecular weight cutoff of $10 \mathrm{kDa}$; Millipore Amicon Ultra-15) followed by resuspension in PBS to a final concentration of $200 \mathrm{mg} \mathrm{mL}^{-1}$. NPs were characterized by dynamic light scattering (DLS) to ensure they passed our QC parameters.

PNP Hydrogel Preparation: PNP hydrogels using 2:10 of HPMC $-\mathrm{C}_{12}$ : PEG-PLA NP were made by mixing a 2:3:1 weight ratio of $6 \mathrm{wt} \% \mathrm{HPMC}-\mathrm{C}_{12}$ polymer solution, $20 \mathrm{wt} \% \mathrm{NP}$ solution, 
and PBS (with or without adjuvants). The NP and aqueous components were loaded into one syringe, the HPMC- $\mathrm{C}_{12}$ was loaded into a second syringe and components were mixed using an elbow connector. After mixing, the elbow was replaced with a 21-gauge needle for injection.

Vaccine Preparation: All the following vaccine variants contained $10 \mu \mathrm{g}$ SARS-CoV-2 RBD per dose. Each bolus vaccine dose $(100 \mu \mathrm{L}$ injection volume) contained $115 \mu \mathrm{g}$ Alhydrogel (Invivogen) in PBS with $10 \mu \mathrm{g}$ or 50 ug 2'3'-cGAMP (Invivogen) added where indicated. PNP hydrogel vaccines were made by diluting respective vaccine cargo in PBS and then mixing a 2:3:1 weight ratio of $6 \mathrm{wt} \%$ HPMC-C12 polymer solution, 20 wt \% NP solution, and PBS (-/+ cargoes). As indicated, each PNP hydrogel dose (150 $\mu \mathrm{L}$ injection volume) contained $115 \mu \mathrm{g}$ Alhydrogel, $10 \mu \mathrm{g}$ or $50 \mu \mathrm{g}$ 2'3'-cGAMP, or $50 \mu \mathrm{g}$ Poly(l:C) (Sigma-Aldrich).

cGAMP In vitro Release Studies: Hydrogels were prepared as described above in the "PNP Hydrogel Preparation" and "Vaccine Preparation" sections. Glass capillary tubes were plugged at one end with epoxy and $150 \mu \mathrm{L}$ of gel was injected into the bottom of 3 separate tubes per treatment. $350 \mu \mathrm{L}$ PBS was added on top of each gel. Tubes were stored upright in an incubator at $37^{\circ} \mathrm{C}$ for about 3 weeks. At each timepoint, $\sim 300 \mu \mathrm{L}$ of PBS was removed and the same amount was replaced. The amount of cGAMP released at each timepoint was determined by measurement of A260 absorbance relative to a standard curve by Nanodrop. The cumulative release was calculated and normalized to the total amount released over the duration of the experiment. Points were fit with the nonlinear "One site - Total fit" model in GraphPad Prism and the half-life was determined.

Mouse immunizations: C57BL/6 (B6) mice were purchased from Charles River and housed at Stanford University. Female mice between 8 and 10 weeks of age at the start of the experiment were used. Mice received a subcutaneous (SC) injection of bolus $(150 \mu \mathrm{L})$ or hydrogel $(150$ $\mu \mathrm{L})$ vaccine on their backs under brief isoflurane anesthesia. PBS injections used a 26-gauge needle, and gel injections used a 21-gauge needle. Blood was collected by tail vein bleeds for 
survival studies or through cardiac puncture for terminal studies, with serum processed using Z gel microtubes (Sarstedt). In the immune infiltration experiment, SC gels were collected for analysis after euthanasia.

Anti-RBD ELISA with mouse serum: MaxiSorp round-bottom immuno 96-well plates (ThermoFisher) were coated with RBD antigen $\left(2 \mu \mathrm{g} \mathrm{mL} \mathrm{m}^{-1}\right.$ in PBS, $\mathrm{pH} 7.4,50 \mu \mathrm{L}$ well $^{-1}$ ) overnight at $4{ }^{\circ} \mathrm{C}$. Additional ELISAs were done with spike and spike mutant-coated plates (wildtype, UK variant B.1.1.7, South Africa variant B.1.351; Sino Biological). After coating, plates were washed 3x (PBS with 0.05\% Tween-20). Plates were blocked with PBS containing $1 \%$ BSA $\left(250 \mu \mathrm{L}\right.$ well $\left.^{-1}\right)$ for $1 \mathrm{~h}$ at RT and then washed $3 \mathrm{x}$. Mouse sera were diluted in diluent buffer (PBS with 1\% BSA and 0.05\% Tween-20) starting at 1:50 and 4-fold serially dilution. A negative reference control consisting of pooled non-immunized sera was included in duplicate on each plate at 1:50 dilution for all determinations. Diluted sera were added to coated/blocked plates $\left(50 \mu \mathrm{L}\right.$ well ${ }^{-1}$ ) and incubated for $2 \mathrm{~h}$ at RT (for total lgG analysis) or overnight at $4{ }^{\circ} \mathrm{C}$ (for $\lg \mathrm{G} 1$ and $\lg \mathrm{G} 2$ analysis). Plates were washed $5 \mathrm{x}$. The respective HRP-conjugated secondaries were diluted in dilution buffer and incubated as follows:

- Total IgG: goat anti-mouse IgG Fc, Invitrogen \#A16084, 1:10,000, $1 \mathrm{~h}$ at RT

- IgG1: goat anti-mouse IgG1 Fc, Abcam \#ab97240, 1:50,000, 2 h at $4{ }^{\circ} \mathrm{C}$

- IgG2c: goat anti-mouse IgG2c Heavy chain, Abcam \#ab97255, 1:10,000, 1 h at RT). ELISA plates were then washed 6x. Plates were developed using high-sensitivity TMB ELISA subtrate (Abcam) for $8 \mathrm{~min}$ at RT and quenched with $1 \mathrm{M} \mathrm{HCl}$. Absorbance at $450 \mathrm{~nm}$ was measured using a Tecan Spark plate reader. The endpoint threshold was set as 2 times the reference negative control average obtained each day. Sample dilution curves were imported into GraphPad Prism 8.4.1, fit with a three-parameter non-linear regression (baseline constrained to 0), and dilution titer value at which the endpoint threshold was crossed for each curve was imputed. Samples failing to meet endpoint threshold at a 1:50 dilution were set to a titer cutoff of 1:25 or below the limit quantitation for the assay. 
Mouse IFN $\alpha$ ELISA: Determination of IFN $\alpha$ concentrations in mouse sera were determined using PBL Assay Science VeriKine-HS Mouse IFN- $\alpha$ All Subtype ELISA Kits. The manufactuter recommended protocol was followed with sera assayed at a 1:20 dilution and then concentrations imputed from a standard curve.

SARS-CoV-2 pseudotyped lentivirus production and viral neutralization assays: SARS-CoV-2 spike pseudotyped lentivirus was produced in HEK293T cells via calcium phosphate transfection. Six million cells were seeded in D10 medium (DMEM + additives: 10\% fetal bovine serum, L-glutamate, penicillin, streptomycin, and $10 \mathrm{mM} \mathrm{HEPES)} \mathrm{in} \mathrm{10-cm} \mathrm{plates} \mathrm{one}$ day prior to transfection. A five-plasmid system was used for viral production consisting of the lentiviral packaging vector (pHAGE_Luc2_IRES_ZsGreen), the SARS-CoV-2 spike, and lentiviral helper plasmids (HDM-Hgpm2, HDM-Tat1b, and pRC-CMV_Rev1b). ${ }^{[42]}$ The spike vector contained the full-length wild-type spike sequence from the Wuhan-Hu-1 strain of SARS-CoV-2 (GenBank NC_045512). Plasmids were added to filter-sterilized water as follows: $10 \mu \mathrm{g}$ pHAGE_Luc2_IRS_ZsGreen, 3.4 $\mu \mathrm{g}$ SARS-CoV-2 spike, $2.2 \mu \mathrm{g}$ HDM-Hgpm2, $2.2 \mu \mathrm{g}$ HDM-Tat1b, and $2.2 \mu \mathrm{g}$ pRC-CMV_Rev1b in a final volume of $500 \mu \mathrm{L}$. HEPES-buffered Saline $(2 \mathrm{X}, \mathrm{pH} 7.0)$ was added dropwise to this mixture to a final volume of $1 \mathrm{~mL}$. To form transfection complexes, $100 \mu \mathrm{L} 2.5 \mathrm{M} \mathrm{CaCl}_{2}$ were added dropwise while the solution was gently agitated. Transfection reactions were incubated for $20 \mathrm{~min}$ at RT, then added dropwise to plated cells. Medium was removed $\sim 24 \mathrm{~h}$ post transfection and replaced with fresh D10 medium. Virus-containing culture supernatants were harvested $\sim 72 \mathrm{~h}$ post transfection via centrifugation at $300 \times \mathrm{g}$ for $5 \mathrm{~min}$ and filtered through a $0.45 \mu \mathrm{m}$ filter. Viral stocks were aliquoted and stored at $-80^{\circ} \mathrm{C}$ until use. For viral neutralization assays, ACE2/HeLa cells were plated in white-walled clear-bottom 96-well plates at 5,000 cells well ${ }^{-1} 1$ day prior to infection. ${ }^{[43]}$ Mouse serum was centrifuged at $2,000 \times \mathrm{g}$ for $15 \mathrm{~min}$, heat inactivated for $30 \mathrm{~min}$ at $56{ }^{\circ} \mathrm{C}$ and diluted in D10 medium. Titered virus was diluted in D10 medium, added to diluted sera, and incubated for $1 \mathrm{~h}$ at $37^{\circ} \mathrm{C}$. Virus:sera dilutions were then transferred to the plated ACE2/HeLa from which seeding media had been aspirated. Polybrene was then spiked in to each well for 
a final concentration of $5 \mu \mathrm{g} \mathrm{mL}{ }^{-1}$ and plates were incubated at $37^{\circ} \mathrm{C}$ for $\sim 48 \mathrm{~h}$. Cells were lysed by adding BriteLite assay readout solution (Perkin Elmer) and luminescence values were measured with a Tecan Spark plate reader. Each plate was normalized by averaging RLUs from wells with cells only ( $0 \%$ infectivity) and virus only (100\% infectivity). Normalized values were fit with a three-parameter non-linear regression inhibitor curve in GraphPad Prism 8.4.1 to obtain $I C_{50}$ values. Fits for all serum neutralization assays were constrained to have a value of $0 \%$ at the bottom of the fit. The limit of quantitation for this assay is approximately $1: 100$ serum dilution. Serum samples that failed to neutralize or that neutralized at levels higher than 1:100 were set at the limit of quantitation.

Flow cytometry analysis of immune cell infiltration: Gels were injected as described in mouse immunizations. After four days, mice were euthanized, and gels, draining inguinal lymph nodes and spleens were excised and placed in $10 \mathrm{~mL}$ of media. Subsequently, gels were placed in a $5 \mathrm{~cm}$ Petri dish and mechanically disrupted between 2 frosted glass slides. The resulting suspension was strained through a $100 \mu \mathrm{M}$ filter into a $50 \mathrm{~mL}$ conical tube and washed $2 \mathrm{x}$ with PBS. Lymph nodes and spleens were incubated in RPMI supplemented with $10 \%$ FBS containing $20 \mu \mathrm{g} / \mathrm{ml}$ DNase I type IV (Sigma-Aldrich) and $1 \mathrm{mg} / \mathrm{ml}$ collagenase from Clostridium histolyticum (Sigma-Aldrich) at $37^{\circ} \mathrm{C}$ for $20 \mathrm{~min}$. Organs were passed through a 100- $\mu \mathrm{m}$ cell strainer (Sigma-Aldrich), and red blood cells were lysed with red blood cell lysis buffer ( $155 \mathrm{mM} \mathrm{NH}_{4} \mathrm{Cl}, 12 \mathrm{mM} \mathrm{NaHCO}_{3}$ and $0.1 \mathrm{mM} \mathrm{EDTA}$ ) for $5 \mathrm{~min}$ at room temperature. Cell pellets (gel, lymph node, and spleen $\sim 200 \mu \mathrm{L}$ in PBS) were then transferred to a 96-well plate for staining. Samples were first stained with LIVE/DEAD Fixable Blue Dead Cell Stain (Invitrogen) for 30 min then fixed and permeabilized with eBioscience Foxp3/Transcription Factor Staining Buffer Set (Invitrogen). Samples were next Fc-blocked for 10 min using TruStain fcX (BioLegend), and then stained for $1 \mathrm{~h}$ (see Supplemental Table S8 for antibodies and dilutions). Flow cytometry analysis for this project was done on an Aurora analyzer (Cytek) in the Stanford Shared FACS Facility. 
Animal Protocol: All animal studies were performed in accordance with National Institutes of Health guidelines and with the approval of the Stanford Administrative Panel on Laboratory Animal Care (Protocol APLAC-32109).

Statistical Analysis: Statistical analyses were conducted using GraphPad Prism. Comparisons of titer data between treatment groups and comparisons of titers to different spike variants within a treatment group were done using 2way ANOVAs with either Dunnett's or Tukey's multiple comparisons test (depending on number of groups compared) on the logged titer values (Figure 2, Table S1-5). Comparisons of $\mathrm{IC}_{50}$ values were done using an Ordinary oneway ANOVA with Dunnett's multiple comparisons test (Figure 3, Table S6). Select P values are shown in the text and remaining $P$ values are in Tables S1-6.

\section{Acknowledgements}

We would like to thank all members of the Appel and Li labs for their useful discussion and advice throughout this project. We want to acknowledge the staff of the BioE/ChemE Animal Facility who cared for our mice. Flow cytometry analysis for this project was done on an Aurora analyzer (Cytek) in the Stanford Shared FACS Facility. This research was financially supported by the Center for Human Systems Immunology with the Bill and Melinda Gates Foundation (OPP1113682; OPP1211043). This work was also supported by the Stanford Maternal and Child Health Research Institute postdoctoral fellowship (to AEP), the Stanford School of Medicine Startup fund (to LL), and Stanford Medical School Discovery Innovation Fund (to LL). We thank Dr. Jesse Bloom, Kate Crawford, Dr. Dennis Burton, and Dr. Deli Huang for sharing the plasmids, cells, and invaluable advice for implementation of the spike-pseudotyped lentiviral neutralization assay. We thank the NIH Cell and Molecular Biology Training Program (T32 GM007276; to ECG) and the Eastman Kodak Fellowship (BSU). 


\section{References}

[1] P. J. Hotez, M. E. Bottazzi, PLoS Negl Trop Dis 2020, 14, e0008548.

[2] W. H. Chen, S. M. Chag, M. V. Poongavanam, A. B. Biter, E. A. Ewere, W. Rezende, C. A. Seid, E. M. Hudspeth, J. Pollet, C. P. McAtee, U. Strych, M. E. Bottazzi, P. J. Hotez, J Pharm Sci 2017, 106, 1961.

[3] V. Vetter, G. Denizer, L. R. Friedland, J. Krishnan, M. Shapiro, Ann Med 2018, 50, 110.

[4] M. Doherty, R. Schmidt-Ott, J. I. Santos, L. R. Stanberry, A. M. Hofstetter, S. L. Rosenthal, A. L. Cunningham, Vaccine 2016, 34, 6681.

[5] D. Stadlbauer, F. Amanat, V. Chromikova, K. Jiang, S. Strohmeier, G. A. Arunkumar, J. Tan, D. Bhavsar, C. Capuano, E. Kirkpatrick, P. Meade, R. N. Brito, C. Teo, M. McMahon, V. Simon, F. Krammer, Curr Protoc Microbiol 2020, 57, e100.

[6] D. Esposito, J. Mehalko, M. Drew, K. Snead, V. Wall, T. Taylor, P. Frank, J. P. Denson, M. Hong, G. Gulten, K. Sadtler, S. Messing, W. Gillette, bioRxiv 2020.

[7] L. Piccoli, Y. J. Park, M. A. Tortorici, N. Czudnochowski, A. C. Walls, M. Beltramello, C. Silacci-Fregni, D. Pinto, L. E. Rosen, J. E. Bowen, O. J. Acton, S. Jaconi, B. Guarino, A. Minola, F. Zatta, N. Sprugasci, J. Bassi, A. Peter, A. De Marco, J. C. Nix, F. Mele, S. Jovic, B. F. Rodriguez, S. V. Gupta, F. Jin, G. Piumatti, G. Lo Presti, A. F. Pellanda, M. Biggiogero, M. Tarkowski, M. S. Pizzuto, E. Cameroni, C. Havenar-Daughton, M. Smithey, D. Hong, V. Lepori, E. Albanese, A. Ceschi, E. Bernasconi, L. Elzi, P. Ferrari, C. Garzoni, A. Riva, G. Snell, F. Sallusto, K. Fink, H. W. Virgin, A. Lanzavecchia, D. Corti, D. Veesler, Cell 2020, 183, 1024.

[8] T. L. Steffen, E. T. Stone, M. Hassert, E. Geerling, B. T. Grimberg, A. M. Espino, P. Pantoja, C. Climent, D. F. Hoft, S. L. George, C. A. Sariol, A. K. Pinto, J. D. Brien, bioRxiv 2020, 2020.08.21.261727.

[9] K. M. Cirelli, D. G. Carnathan, B. Nogal, J. T. Martin, O. L. Rodriguez, A. A. Upadhyay, C. A. Enemuo, E. H. Gebru, Y. Choe, F. Viviano, C. Nakao, M. G. Pauthner, S. Reiss, C. A. Cottrell, M. L. Smith, R. Bastidas, W. Gibson, A. N. Wolabaugh, M. B. Melo, B. Cossette, V. Kumar, N. B. Patel, T. Tokatlian, S. Menis, D. W. Kulp, D. R. Burton, B. Murrell, W. R. Schief, S. E. Bosinger, A. B. Ward, C. T. Watson, G. Silvestri, D. J. Irvine, S. Crotty, Cell 2019, 177, 1153; Z. B. Wang, J. Xu, Vaccines (Basel) 2020, 8.

[10] A. Ablasser, J. L. Schmid-Burgk, I. Hemmerling, G. L. Horvath, T. Schmidt, E. Latz, V. Hornung, Nature 2013, 503, 530.

[11] W. R. Heath, Y. Kato, T. M. Steiner, I. Caminschi, Curr Opin Immunol 2019, 58, 44.

[12] L. Li, Q. Yin, P. Kuss, Z. Maliga, J. L. Millan, H. Wu, T. J. Mitchison, Nat Chem Biol 2014, 10, 1043.

[13] A. F. Cordova, C. Ritchie, V. Böhnert, L. Li, ACS Central Science 2021.

[14] X. D. Li, J. Wu, D. Gao, H. Wang, L. Sun, Z. J. Chen, Science 2013, 341, 1390.

[15] J. A. Carozza, V. Bohnert, K. C. Nguyen, G. Skariah, K. E. Shaw, J. A. Brown, M. Rafat, R. von Eyben, E. E. Graves, J. S. Glenn, M. Smith, L. Li, Nat Cancer 2020, 1, 184.

[16] L. Motedayen Aval, J. E. Pease, R. Sharma, D. J. Pinato, J Clin Med 2020, 9.

[17] F. Meric-Bernstam, S. K. Sandhu, O. Hamid, A. Spreafico, S. Kasper, R. Dummer, T. Shimizu, N. Steeghs, N. Lewis, C. C. Talluto, S. Dolan, A. Bean, R. Brown, D. Trujillo, N. Nair, J. J. Luke, Journal of Clinical Oncology 2019, 37, 2507.

[18] T. W. Dubensky, Jr., D. B. Kanne, M. L. Leong, Ther Adv Vaccines 2013, 1, 131.

[19] H. Gogoi, S. Mansouri, L. Jin, Vaccines (Basel) 2020, 8. 
[20] D. Shae, J. J. Baljon, M. Wehbe, P. P. Christov, K. W. Becker, A. Kumar, N. Suryadevara, C. S. Carson, C. R. Palmer, F. C. Knight, S. Joyce, J. T. Wilson, ACS Nano 2020, 14, 9904.

[21] M. A. Collier, R. D. Junkins, M. D. Gallovic, B. M. Johnson, M. M. Johnson, A. N. Macintyre, G. D. Sempowski, E. M. Bachelder, J. P. Ting, K. M. Ainslie, Mol Pharm 2018, 15, 4933.

[22] T. J. Goodwin, L. Huang, Vaccine 2017, 35, 2550.

[23] E. Lee, H. E. Jang, Y. Y. Kang, J. Kim, J. H. Ahn, H. Mok, Acta Biomater 2016, 29, 271.

[24] J. Wang, P. Li, Y. Yu, Y. Fu, H. Jiang, M. Lu, Z. Sun, S. Jiang, L. Lu, M. X. Wu, Science 2020, 367.

[25] C. Ritchie, A. F. Cordova, G. T. Hess, M. C. Bassik, L. Li, Mol Cell 2019, 75, 372.

[26] R. D. Luteijn, S. A. Zaver, B. G. Gowen, S. K. Wyman, N. E. Garelis, L. Onia, S. M. McWhirter, G. E. Katibah, J. E. Corn, J. J. Woodward, D. H. Raulet, Nature 2019, 573, 434.

[27] L. J. Lahey, R. E. Mardjuki, X. Wen, G. T. Hess, C. Ritchie, J. A. Carozza, V. Bohnert, M. Maduke, M. C. Bassik, L. Li, Mol Cell 2020, 80, 578.

[28] C. Zhou, X. Chen, R. Planells-Cases, J. Chu, L. Wang, L. Cao, Z. Li, K. I. LopezCayuqueo, Y. Xie, S. Ye, X. Wang, F. Ullrich, S. Ma, Y. Fang, X. Zhang, Z. Qian, X. Liang, S. Q. Cai, Z. Jiang, D. Zhou, Q. Leng, T. S. Xiao, K. Lan, J. Yang, H. Li, C. Peng, Z. Qiu, T. J. Jentsch, H. Xiao, Immunity 2020, 52, 767.

[29] G. A. Roth, E. C. Gale, M. Alcántara-Hernández, W. Luo, E. Axpe, R. Verma, Q. Yin, A. C. Yu, H. Lopez Hernandez, C. L. Maikawa, A. A. A. Smith, M. M. Davis, B. Pulendran, J. Idoyaga, E. A. Appel, ACS Central Science 2020.

[30] E. C. Gale, A. E. Powell, G. A. Roth, B. S. Ou, E. L. Meany, A. K. Grosskopf, J. Adamska, V. Picece, A. I. d'Aquino, B. Pulendran, P. S. Kim, E. A. Appel, bioRxiv 2021.

[31] D. Gao, J. Wu, Y. T. Wu, F. Du, C. Aroh, N. Yan, L. Sun, Z. J. Chen, Science 2013, $341,903$.

[32] M. Jeyanathan, S. Afkhami, F. Smaill, M. S. Miller, B. D. Lichty, Z. Xing, Nat Rev Immunol 2020, 20, 615.

[33] H. Lopez Hernandez, J. W. Souza, E. A. Appel, Macromol Biosci 2021, 21, e2000295.

[34] G. A. Roth, O. M. Saouaf, A. A. A. Smith, E. C. Gale, M. A. Hernandez, J. Idoyaga, E. A. Appel, ACS Biomater Sci Eng 2021.

[35] J. A. Aebig, G. E. Mullen, G. Dobrescu, K. Rausch, L. Lambert, O. Ajose-Popoola, C. A. Long, A. Saul, A. P. Miles, J Immunol Methods 2007, 323, 139.

[36] N. A. Peppas, J Control Release 2014, 190, 31.

[37] A. Murira, A. Lamarre, Front Immunol 2016, 7, 609.

[38] J. Fornefett, J. Krause, K. Klose, F. Fingas, R. Hassert, L. Benga, T. Grunwald, U. Muller, W. Schrodl, C. G. Baums, BMC Microbiol 2018, 18, 45.

[39] E. Oleszycka, E. C. Lavelle, Curr Opin Immunol 2014, 28, 1.

[40] R. D. Junkins, M. D. Gallovic, B. M. Johnson, M. A. Collier, R. Watkins-Schulz, N. Cheng, C. N. David, C. E. McGee, G. D. Sempowski, I. Shterev, K. McKinnon, E. M. Bachelder, K. M. Ainslie, J. P. Ting, J Control Release 2018, 270, 1.

[41] A. Fontanet, B. Autran, B. Lina, M. P. Kieny, S. S. A. Karim, D. Sridhar, Lancet 2021. 
[42] K. H. D. Crawford, R. Eguia, A. S. Dingens, A. N. Loes, K. D. Malone, C. R. Wolf, H. Y. Chu, M. A. Tortorici, D. Veesler, M. Murphy, D. Pettie, N. P. King, A. B. Balazs, J. D. Bloom, Viruses 2020, 12.

[43] T. F. Rogers, F. Zhao, D. Huang, N. Beutler, A. Burns, W. T. He, O. Limbo, C. Smith, G. Song, J. Woehl, L. Yang, R. K. Abbott, S. Callaghan, E. Garcia, J. Hurtado, M. Parren, L. Peng, S. Ramirez, J. Ricketts, M. J. Ricciardi, S. A. Rawlings, N. C. Wu, M. Yuan, D. M. Smith, D. Nemazee, J. R. Teijaro, J. E. Voss, I. A. Wilson, R. Andrabi, B. Briney, E. Landais, D. Sok, J. G. Jardine, D. R. Burton, Science 2020, 369, 956.

[44] A. E. Powell, K. Zhang, M. Sanyal, S. Tang, P. A. Weidenbacher, S. Li, T. D. Pham, J. E. Pak, W. Chiu, P. S. Kim, bioRxiv 2020.

[45] D. M. Hinton, Convalescent Plasma EUA Letter of Authorization. (March 9, 2021).

[46] D. Ellis, N. Brunette, K. H. D. Crawford, A. C. Walls, M. N. Pham, C. Chen, K.-L. Herpoldt, B. Fiala, M. Murphy, D. Pettie, J. C. Kraft, K. D. Malone, M. J. Navarro, C. Ogohara, E. Kepl, R. Ravichandran, C. Sydeman, M. Ahlrichs, M. Johnson, A. Blackstone, L. Carter, T. N. Starr, A. J. Greaney, K. K. Lee, D. Veesler, J. D. Bloom, N. P. King, bioRxiv 2021, 2021.05.15.444222.

[47] N. C. Dalvie, S. A. Rodriguez-Aponte, B. L. Hartwell, L. H. Tostanoski, A. M. Biedermann, L. E. Crowell, K. Kaur, O. Kumru, L. Carter, J. Yu, A. Chang, K. McMahan, T. Courant, C. Lebas, A. A. Lemnios, K. A. Rodrigues, M. Silva, R. S. Johnston, C. A. Naranjo, M. K. Tracey, J. R. Brady, C. A. Whittaker, D. Yun, S. Kar, M. Porto, M. Lok, H. Andersen, M. G. Lewis, K. R. Love, D. L. Camp, J. M. Silverman, H. Kleanthous, S. B. Joshi, D. B. Volkin, P. M. Dubois, N. Collin, N. P. King, D. H. Barouch, D. J. Irvine, J. C. Love, bioRxiv 2021, 2021.03.03.433558.

[48] M. Merad, P. Sathe, J. Helft, J. Miller, A. Mortha, Annu Rev Immunol 2013, 31, 563.

[49] A. Mildner, S. Jung, Immunity 2014, 40, 642.

[50] E. Segura, S. Amigorena, Trends Immunol 2013, 34, 440.

[51] A. C. Walls, B. Fiala, A. Schafer, S. Wrenn, M. N. Pham, M. Murphy, L. V. Tse, L. Shehata, M. A. O'Connor, C. Chen, M. J. Navarro, M. C. Miranda, D. Pettie, R. Ravichandran, J. C. Kraft, C. Ogohara, A. Palser, S. Chalk, E. C. Lee, K. Guerriero, E. Kepl, C. M. Chow, C. Sydeman, E. A. Hodge, B. Brown, J. T. Fuller, K. H. Dinnon, 3rd, L. E. Gralinski, S. R. Leist, K. L. Gully, T. B. Lewis, M. Guttman, H. Y. Chu, K. K. Lee, D. H. Fuller, R. S. Baric, P. Kellam, L. Carter, M. Pepper, T. P. Sheahan, D. Veesler, N. P. King, Cell 2020, 183, 1367.

[52] H. L. Janice Oh, S. Ken-En Gan, A. Bertoletti, Y. J. Tan, Emerg Microbes Infect 2012, 1 , e23.

[53] M. Matsumoto, T. Seya, Adv. Drug Deliv. Rev. 2008, 60, 805; E. C. Gale, G. A. Roth, A. A. A. Smith, M. Alcántara-Hernández, J. Idoyaga, E. A. Appel, Advanced Therapeutics 2020, 3, 1900174.

[54] F. Amanat, D. Stadlbauer, S. Strohmeier, T. H. O. Nguyen, V. Chromikova, M. McMahon, K. Jiang, G. Asthagiri Arunkumar, D. Jurczyszak, J. Polanco, M. BermudezGonzalez, G. Kleiner, T. Aydillo, L. Miorin, D. Fierer, L. Amarilis Lugo, E. Milunka Kojic, J. Stoever, S. T. H. Liu, C. Cunningham-Rundles, P. L. Felgner, T. Moran, A. GarciaSastre, D. Caplivski, A. Cheng, K. Kedzierska, O. Vapalahti, J. M. Hepojoki, V. Simon, F. Krammer, medRxiv 2020.

[55] E. A. Appel, M. W. Tibbitt, M. J. Webber, B. A. Mattix, O. Veiseh, R. Langer, Nat Commun 2015, 6, 6295.

[56] A. B. Vogel, I. Kanevsky, Y. Che, K. A. Swanson, A. Muik, M. Vormehr, L. M. Kranz, K. C. Walzer, S. Hein, A. Guler, J. Loschko, M. S. Maddur, A. Ota-Setlik, K. Tompkins, J. Cole, B. G. Lui, T. Ziegenhals, A. Plaschke, D. Eisel, S. C. Dany, S. Fesser, S. Erbar, 
F. Bates, D. Schneider, B. Jesionek, B. Sanger, A. K. Wallisch, Y. Feuchter, H. Junginger, S. A. Krumm, A. P. Heinen, P. Adams-Quack, J. Schlereth, S. Schille, C. Kroner, R. de la Caridad Guimil Garcia, T. Hiller, L. Fischer, R. S. Sellers, S. Choudhary, O. Gonzalez, F. Vascotto, M. R. Gutman, J. A. Fontenot, S. Hall-Ursone, K. Brasky, M. C. Griffor, S. Han, A. A. H. Su, J. A. Lees, N. L. Nedoma, E. H. Mashalidis, P. V. Sahasrabudhe, C. Y. Tan, D. Pavliakova, G. Singh, C. Fontes-Garfias, M. Pride, I. L. Scully, T. Ciolino, J. Obregon, M. Gazi, R. Carrion, Jr., K. J. Alfson, W. V. Kalina, D. Kaushal, P. Y. Shi, T. Klamp, C. Rosenbaum, A. N. Kuhn, O. Tureci, P. R. Dormitzer, K. U. Jansen, U. Sahin, Nature 2021, 592, 283.

[57] L. R. Volpatti, R. P. Wallace, S. Cao, M. M. Raczy, R. Wang, L. T. Gray, A. T. Alpar, P. S. Briquez, N. Mitrousis, T. M. Marchell, M. S. Sasso, M. Nguyen, A. Mansurov, E. Budina, A. Solanki, E. A. Watkins, M. R. Schnorenberg, A. C. Tremain, J. W. Reda, V. Nicolaescu, K. Furlong, S. Dvorkin, S. S. Yu, B. Manicassamy, J. L. LaBelle, M. V. Tirrell, G. Randall, M. Kwissa, M. A. Swartz, J. A. Hubbell, bioRxiv 2021, 2021.04.08.438884. 